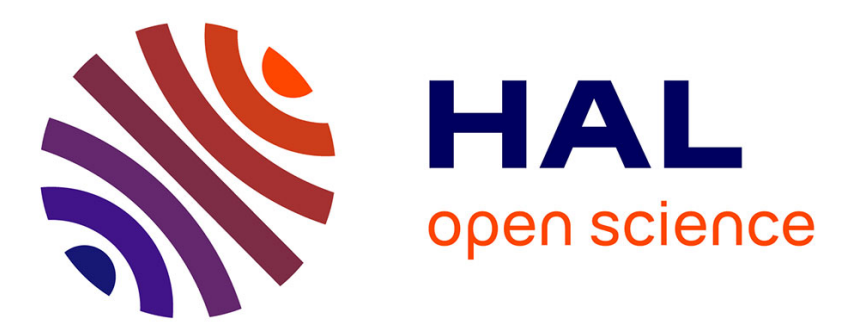

\title{
FROM STRONG TO VERY WEAK SOLUTIONS TO STOKES SYSTEM WITH NAVIER BOUNDARY CONDITIONS IN THE HALF-SPACE
}

Chérif Amrouche, Sárka Necasová, Yves Raudin

\section{> To cite this version:}

Chérif Amrouche, Sárka Necasová, Yves Raudin. FROM STRONG TO VERY WEAK SOLUTIONS TO STOKES SYSTEM WITH NAVIER BOUNDARY CONDITIONS IN THE HALF-SPACE. SIAM Journal on Mathematical Analysis, 2009, 41 (5), pp.1792-1815. hal-00428966

\section{HAL Id: hal-00428966 https://hal.science/hal-00428966}

Submitted on 30 Oct 2009

HAL is a multi-disciplinary open access archive for the deposit and dissemination of scientific research documents, whether they are published or not. The documents may come from teaching and research institutions in France or abroad, or from public or private research centers.
L'archive ouverte pluridisciplinaire HAL, est destinée au dépôt et à la diffusion de documents scientifiques de niveau recherche, publiés ou non, émanant des établissements d'enseignement et de recherche français ou étrangers, des laboratoires publics ou privés. 


\title{
FROM STRONG TO VERY WEAK SOLUTIONS TO STOKES SYSTEM WITH NAVIER BOUNDARY CONDITIONS IN THE HALF-SPACE
}

\author{
CHÉRIF AMROUCHE ${ }^{\dagger}$, ŠÁRKA NEČASOVÁ ${ }^{\ddagger}$, AND YVES RAUDIN ${ }^{\dagger}$
}

\begin{abstract}
We consider the Stokes problem with slip type boundary conditions in the half-space $\mathbb{R}_{+}^{n}$, with $n \geqslant 2$. The weighted Sobolev spaces yield the functional framework. We study generalized and strong solutions and then the case with very low regularity of data on the boundary. We apply the method of decomposition introduced in our previous work (see [7]), where it is necessary to solve particular problems for harmonic and biharmonic operators with very weak data. We also envisage a wide class of behaviour at infinity for data and solutions.
\end{abstract}

Key words. Stokes problem, Half-space, Weighted Sobolev spaces

AMS subject classifications. 35J50, 35J55, 35Q30, 76D07, 76N10

1. Introduction and preliminaries. The motion of a viscous incompressible fluid is described by the Navier-Stokes equations, which are non-linear. The Stokes system is a linear approximation of this model, available for slow motions. For the stationary Stokes problem

$$
-\Delta \boldsymbol{u}+\nabla \pi=\boldsymbol{f} \text { and } \operatorname{div} \boldsymbol{u}=h \text { in } \Omega,
$$

where $\Omega$ is a domain of $\mathbb{R}^{n}$, there are several possibilities of boundary conditions. Under the hypothesis of impermeability of the boundary, the velocity field $\boldsymbol{u}$ satisfies

$$
\boldsymbol{u} \cdot \boldsymbol{n}=0 \text { on } \partial \Omega,
$$

where $\boldsymbol{n}$ stands for the outer normal vector. According to the idea that the fluid cannot slip on the wall due to its viscosity, we get the no-slip condition

$$
\boldsymbol{u}_{\tau}=\mathbf{0} \text { on } \partial \Omega \text {, }
$$

where $\boldsymbol{u}_{\tau}=\boldsymbol{u}-(\boldsymbol{u} \cdot \boldsymbol{n}) \boldsymbol{n}$ denotes as usual the tangential component of $\boldsymbol{u}$. The Dirichlet boundary value problem, which was suggested by Stokes, is the combination of (1.1) and (1.2). Concerning this problem, the bibliography is well known and extensive. Especially in the case of the half-space, we would like to mention the works of Cattabriga [12], Tanaka [25], Farwig and Sohr [15] and Galdi [16] where the solution of problem is investigated in homogeneous Sobolev spaces. Whereas in the works of Maz'ya et al. [21] and Boulmezaoud [11], we can found results in weighted Sobolev spaces. This is also the functional framework of our previous work (see [7]).

The correctness of the no-slip hypothesis has been subjected to discussion for over two centuries by many distinguished scientists. Instead of (1.2), Navier had already proposed a condition saying that the velocity on the boundary is proportional to the tangential component of the stress:

$$
(\mathbb{T} \cdot \boldsymbol{n})_{\tau}+\beta \boldsymbol{u}_{\tau}=\mathbf{0} \text { on } \partial \Omega,
$$

\footnotetext{
†Laboratoire de Mathématiques Appliquées, CNRS UMR 5142, Université de Pau et des Pays de l'Adour, IPRA, Avenue de l'Université, 64000 Pau, France (cherif.amrouche@univ-pau.fr and yves.raudin@univ-pau.fr).

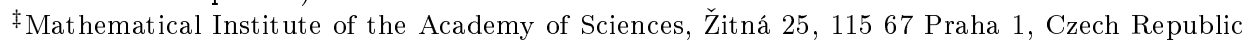
(matus@math. cas.cz).
} 
where $\mathbb{T}$ denotes the viscous stress tensor and $\beta$ is a friction coefficient. For the incompressible isotropic fluids the viscous stress tensor is given by

$$
\mathbb{T}=-\pi \mathbb{I}+\nu\left(\nabla \boldsymbol{u}+\nabla \boldsymbol{u}^{T}\right) .
$$

The case $\beta=0$ is termed complete slip, while (1.3) reduces to (1.2) in the asymptotic limit $\beta \rightarrow \infty$.

Recent developments in micro and nanofluidic technologies have renewed interest in the influence of surface roughness on the slip behaviour of viscous fluids (see Priezjev and Troian [23]). Intuitively much closer to the observed reality, the Navier slip conditions have been often replaced by (1.2) as the slip length is likely to be too small to influence the motion on the macroscopic scale. However, numerous experiments and simulations as well as theoretical studies have shown that the classical no-slip assumption can fail when the walls are sufficiently smooth (see Einzel et al. [13], Lauga et al. [20], Priezjev et al. [22], Qian et al. [24], Zhu and Granick [27]). Strictly speaking, the slip length characterizing the contact between a fluid and a solid wall in relative motion is influenced by many different factors, among which the intrinsic affinity and commensurability between the liquid and solid molecular size as well as the macroscopic surface roughness caused by imperfections and tiny asperities play a significant role. Navier's boundary conditions have been considered by many authors. Let us quote Jäger and Mikelić [18] and Zajączkowski [26]. In the three dimensional case, we can find other boundary conditions in the work of Ladyzhenskaya and Solonnikov [19] and then intensively studied by Babin, Mahalov and Nicolaenko $[8,9,10]$. These conditions can be expressed by (1.1) combined with the equations

$$
\operatorname{curl} \boldsymbol{u} \times \boldsymbol{n}=\mathbf{0},
$$

In the half space $\mathbb{R}_{+}^{n}$, where $\boldsymbol{n}=(0, \ldots, 0,-1)$, Navier's conditions (1.1) and (1.3) with $\beta=0$ can be written in

$$
u_{n}=0, \quad \partial_{n} \boldsymbol{u}^{\prime}=\mathbf{0} \quad \text { on } \Gamma=\partial \mathbb{R}_{+}^{n} .
$$

Let us remark that in the case of $\mathbb{R}_{+}^{3}$, we would get the same boundary conditions from (1.1) and (1.4).

The aim of this paper is to investigate the Stokes problem in the half-space with this type of slip boundary conditions:

$$
\left(S^{\sharp}\right)\left\{\begin{array}{rlll}
-\Delta \boldsymbol{u}+\nabla \pi=\boldsymbol{f} & \text { and } & \operatorname{div} \boldsymbol{u}=h & \text { in } \mathbb{R}_{+}^{n}, \\
u_{n}=g_{n} & \text { and } & \partial_{n} \boldsymbol{u}^{\prime}=\boldsymbol{g}^{\prime} & \text { on } \Gamma .
\end{array}\right.
$$

This paper is organized as follows. The second part of this section is devoted to the notations, functional setting and useful results. In Section 2, we establish the existence of generalized solutions in the central case of weight zero. In Section 3, we extend this result to a wide class of weights and we also deal with strong solutions. Last, in Section 4, we are interested in the case of very low regularity at the boundary which yields very weak solutions.

For any real number $p>1$, we always take $p^{\prime}$ to be the Hölder conjugate of $p$, that is $\frac{1}{p}+\frac{1}{p^{\prime}}=1$.

For any integer $n \geqslant 2$, writing a typical point $x \in \mathbb{R}^{n}$ as $x=\left(x^{\prime}, x_{n}\right)$, we denote by $\mathbb{R}_{+}^{n}$ the upper half-space of $\mathbb{R}^{n}$ and $\Gamma \equiv \mathbb{R}^{n-1}$ its boundary. We will use the two 
basic weights $\varrho=\left(1+|x|^{2}\right)^{1 / 2}$ and $\lg \varrho=\ln \left(2+|x|^{2}\right)$, where $|x|$ is the Euclidean norm of $x$.

For any integer $q, \mathcal{P}_{q}$ stands for the space of polynomials of degree smaller than or equal to $q ; \mathcal{P}_{q}^{\Delta}$ (resp. $\mathcal{P}_{q}^{\Delta^{2}}$ ) is the subspace of harmonic (resp. biharmonic) polynomials of $\mathcal{P}_{q} ; \mathcal{A}_{q}^{\Delta}$ (resp. $\mathcal{N}_{q}^{\Delta}$ ) is the subspace of polynomials of $\mathcal{P}_{q}^{\Delta}$, odd (resp. even) with respect to $x_{n}$, or equivalently, which satisfy the condition $\varphi\left(x^{\prime}, 0\right)=0$ (resp. $\partial_{n} \varphi\left(x^{\prime}, 0\right)=0$ ); with the convention that these spaces are reduced to $\{0\}$ if $q<0$. For any real number $s$, we denote by $[s]$ the integer part of $s$.

Given a Banach space $B$, with dual space $B^{\prime}$ and a closed subspace $X$ of $B$, we denote by $B^{\prime} \perp X$ the subspace of $B^{\prime}$ orthogonal to $X$. For any $k \in \mathbb{Z}$, we will denote by $\{1, \ldots, k\}$ the set of the first $k$ positive integers, with the convention that this set is empty if $k$ is nonpositive.

In the whole text, bold characters are used for the vector fields - depending on the context, $\boldsymbol{f} \in \boldsymbol{X}$ stands for $\boldsymbol{f}=\left(f_{1}, \ldots, f_{n}\right) \in \boldsymbol{X}=X^{n}$ and $\boldsymbol{g}^{\prime} \in \boldsymbol{X}$ stands for $\boldsymbol{g}^{\prime}=\left(g_{1}, \ldots, g_{n-1}\right) \in \boldsymbol{X}=X^{n-1}-$.

About weighted Sobolev spaces, we refer the reader to Hanouzet's classical article [17] and more especially to [2] for logarithmic weights. Let $\Omega$ be an open set of $\mathbb{R}^{n}$. For any $m \in \mathbb{N}, p \in] 1, \infty\left[,(\alpha, \beta) \in \mathbb{R}^{2}\right.$, we define the following space:

$$
\begin{array}{r}
W_{\alpha, \beta}^{m, p}(\Omega)=\left\{u \in \mathcal{D}^{\prime}(\Omega) ; 0 \leqslant|\lambda| \leqslant k, \varrho^{\alpha-m+|\lambda|}(\lg \varrho)^{\beta-1} \partial^{\lambda} u \in L^{p}(\Omega)\right. \\
\left.k+1 \leqslant|\lambda| \leqslant m, \varrho^{\alpha-m+|\lambda|}(\lg \varrho)^{\beta} \partial^{\lambda} u \in L^{p}(\Omega)\right\},
\end{array}
$$

where $k=m-n / p-\alpha$ if $n / p+\alpha \in\{1, \ldots, m\}$, and $k=-1$ otherwise. In the case $\beta=0$, we simply denote the space by $W_{\alpha}^{m, p}(\Omega)$. Note that $W_{\alpha, \beta}^{m, p}(\Omega)$ is a reflexive Banach space equipped with its natural norm:

$$
\begin{aligned}
\|u\|_{W_{\alpha, \beta}^{m, p}(\Omega)}=\left(\sum_{0 \leqslant|\lambda| \leqslant k}\left\|\varrho^{\alpha-m+|\lambda|}(\lg \varrho)^{\beta-1} \partial^{\lambda} u\right\|_{L^{p}(\Omega)}^{p}\right. & \\
& \left.+\sum_{k+1 \leqslant|\lambda| \leqslant m}\left\|\varrho^{\alpha-m+|\lambda|}(\lg \varrho)^{\beta} \partial^{\lambda} u\right\|_{L^{p}(\Omega)}^{p}\right)^{1 / p} .
\end{aligned}
$$

We also define the semi-norm:

$$
|u|_{W_{\alpha, \beta}^{m, p}(\Omega)}=\left(\sum_{|\lambda|=m}\left\|\varrho^{\alpha}(\lg \varrho)^{\beta} \partial^{\lambda} u\right\|_{L^{p}(\Omega)}^{p}\right)^{1 / p} .
$$

The weights in the definition (1.5) are chosen so that $\mathcal{D}\left(\overline{\mathbb{R}_{+}^{n}}\right)$ is dense in $W_{\alpha, \beta}^{m, p}\left(\mathbb{R}_{+}^{n}\right)$ and so that the following Poincaré-type inequality holds in $W_{\alpha, \beta}^{m, p}\left(\mathbb{R}_{+}^{n}\right)$ (see [3]): Let $q^{*}=\inf (q, m-1)$, where $q$ is the highest degree of the polynomials contained in $W_{\alpha, \beta}^{m, p}\left(\mathbb{R}_{+}^{n}\right)$. If $n / p+\alpha \notin\{1, \ldots, m\}$ or $(\beta-1) p \neq-1$, then

$$
\forall u \in W_{\alpha, \beta}^{m, p}\left(\mathbb{R}_{+}^{n}\right), \quad\|u\|_{W_{\alpha, \beta}^{m, p}\left(\mathbb{R}_{+}^{n}\right) / \mathcal{P}_{q^{*}}} \leqslant C|u|_{W_{\alpha, \beta}^{m, p}\left(\mathbb{R}_{+}^{n}\right)},
$$

and

$$
\forall u \in \stackrel{\circ}{\stackrel{\circ}{W}_{\alpha, \beta}^{m, p}}\left(\mathbb{R}_{+}^{n}\right)=\overline{\mathcal{D}\left(\mathbb{R}_{+}^{n}\right)}\|\cdot\|_{W_{\alpha, \beta}^{m, p}\left(\mathbb{R}_{+}^{n}\right)}, \quad\|u\|_{W_{\alpha, \beta}^{m, p}\left(\mathbb{R}_{+}^{n}\right)} \leqslant C|u|_{W_{\alpha, \beta}^{m, p}\left(\mathbb{R}_{+}^{n}\right)} .
$$

We denote by $W_{-\alpha,-\beta}^{-m, p^{\prime}}\left(\mathbb{R}_{+}^{n}\right)$ the dual space of $\stackrel{\circ}{W}_{\alpha, \beta}^{m, p}\left(\mathbb{R}_{+}^{n}\right)$ and we notice that it is a space of distributions. If $n / p+\alpha \notin\{1, \ldots, m\}$, we have the imbeddings:

$$
W_{\alpha, \beta}^{m, p}\left(\mathbb{R}_{+}^{n}\right) \hookrightarrow W_{\alpha-1, \beta}^{m-1, p}\left(\mathbb{R}_{+}^{n}\right) \hookrightarrow \cdots \hookrightarrow W_{\alpha-m, \beta}^{0, p}\left(\mathbb{R}_{+}^{n}\right) .
$$


If $n / p+\alpha=j \in\{1, \ldots, m\}$, then we have:

$$
W_{\alpha, \beta}^{m, p} \hookrightarrow \cdots \hookrightarrow W_{\alpha-j+1, \beta}^{m-j+1, p} \hookrightarrow W_{\alpha-j, \beta-1}^{m-j, p} \hookrightarrow \cdots \hookrightarrow W_{\alpha-m, \beta-1}^{0, p} .
$$

In order to define the traces of functions of $W_{\alpha}^{m, p}\left(\mathbb{R}_{+}^{n}\right)$ (here we don't consider the case $\beta \neq 0$ ), for any $\sigma \in] 0,1[$, we introduce the space:

$$
\begin{aligned}
W_{\alpha}^{\sigma, p}\left(\mathbb{R}^{n}\right)=\left\{u \in \mathcal{D}^{\prime}\left(\mathbb{R}^{n}\right) ; w^{\alpha-\sigma} u \in L^{p}\left(\mathbb{R}^{n}\right),\right. \\
\left.\qquad \int_{\mathbb{R}^{n} \times \mathbb{R}^{n}} \frac{\left|\varrho^{\alpha}(x) u(x)-\varrho^{\alpha}(y) u(y)\right|^{p}}{|x-y|^{n+\sigma p}} \mathrm{~d} x \mathrm{~d} y<\infty\right\},
\end{aligned}
$$

where $w=\varrho$ if $n / p+\alpha \neq \sigma$ and $w=\varrho(\lg \varrho)^{1 /(\sigma-\alpha)}$ if $n / p+\alpha=\sigma$. For any $s \in \mathbb{R}^{+}$, we set

$$
\begin{aligned}
W_{\alpha}^{s, p}\left(\mathbb{R}^{n}\right)= & \left\{u \in \mathcal{D}^{\prime}\left(\mathbb{R}^{n}\right) ; 0 \leqslant|\lambda| \leqslant k, \varrho^{\alpha-s+|\lambda|}(\lg \varrho)^{-1} \partial^{\lambda} u \in L^{p}\left(\mathbb{R}^{n}\right)\right. \\
& \left.k+1 \leqslant|\lambda| \leqslant[s]-1, \varrho^{\alpha-s+|\lambda|} \partial^{\lambda} u \in L^{p}\left(\mathbb{R}^{n}\right) ; \partial^{[s]} u \in W_{\alpha}^{\sigma, p}\left(\mathbb{R}^{n}\right)\right\},
\end{aligned}
$$

where $k=s-n / p-\alpha$ if $n / p+\alpha \in\{\sigma, \ldots, \sigma+[s]\}$, with $\sigma=s-[s]$ and $k=-1$ otherwise. In the same way, we define, for any real number $\beta$, the space $W_{\alpha, \beta}^{s, p}\left(\mathbb{R}^{n}\right)=$ $\left\{v \in \mathcal{D}^{\prime}\left(\mathbb{R}^{n}\right) ;(\lg \varrho)^{\beta} v \in W_{\alpha}^{s, p}\left(\mathbb{R}^{n}\right)\right\}$. They also are reflexive Banach spaces equipped with their naturall norms. If $n / p+\alpha \notin\{\sigma, \ldots, \sigma+[s]-1\}$, we have the imbeddings:

$$
\begin{gathered}
W_{\alpha, \beta}^{s, p}\left(\mathbb{R}^{n}\right) \hookrightarrow W_{\alpha-1, \beta}^{s-1, p}\left(\mathbb{R}^{n}\right) \hookrightarrow \cdots \hookrightarrow W_{\alpha-[s], \beta}^{\sigma, p}\left(\mathbb{R}^{n}\right), \\
W_{\alpha, \beta}^{s, p}\left(\mathbb{R}^{n}\right) \hookrightarrow W_{\alpha+[s]-s, \beta}^{[s], p}\left(\mathbb{R}^{n}\right) \hookrightarrow \cdots \hookrightarrow W_{\alpha-s, \beta}^{0, p}\left(\mathbb{R}^{n}\right) .
\end{gathered}
$$

If $n / p+\alpha=j \in\{\sigma, \ldots, \sigma+[s]-1\}$, then we have:

$$
\begin{gathered}
W_{\alpha, \beta}^{s, p} \hookrightarrow \cdots \hookrightarrow W_{\alpha-j+1, \beta}^{s-j+1, p} \hookrightarrow W_{\alpha-j, \beta-1}^{s-j, p} \hookrightarrow \cdots \hookrightarrow W_{\alpha-[s], \beta-1}^{\sigma, p}, \\
W_{\alpha, \beta}^{s, p} \hookrightarrow W_{\alpha+[s]-s, \beta}^{[s], p} \hookrightarrow \cdots \hookrightarrow W_{\alpha-\sigma-j+1, \beta}^{[s]-j+1, p} \hookrightarrow W_{\alpha-\sigma-j, \beta-1}^{[s]-j, p} \hookrightarrow \cdots \hookrightarrow W_{\alpha-s, \beta-1}^{0, p} .
\end{gathered}
$$

If $u$ is a function on $\mathbb{R}_{+}^{n}$, we denote its trace of order $j$ on the hyperplane $\Gamma$ by:

$$
\forall j \in \mathbb{N}, \quad \gamma_{j} u: x^{\prime} \in \mathbb{R}^{n-1} \longmapsto \partial_{n}^{j} u\left(x^{\prime}, 0\right) .
$$

Let us recall the following trace lemma due to Hanouzet (see [17]) and extended by Amrouche and Nečasová (see [3]) to the critical values with logarithmic weights:

Lemma 1.1 (The Trace Lemma). For any integer $m \geqslant 1$ and real number $\alpha$, we have the linear continuous mapping

$$
\gamma=\left(\gamma_{0}, \gamma_{1}, \ldots, \gamma_{m-1}\right): W_{\alpha}^{m, p}\left(\mathbb{R}_{+}^{n}\right) \longrightarrow \prod_{j=0}^{m-1} W_{\alpha}^{m-j-1 / p, p}\left(\mathbb{R}^{n-1}\right)
$$

Moreover $\gamma$ is surjective and $\operatorname{Ker} \gamma=\stackrel{\circ}{W_{\alpha}^{m}}, p\left(\mathbb{R}_{+}^{n}\right)$.

On the Stokes problem in $\mathbb{R}^{n}$

$$
(S): \quad-\Delta \boldsymbol{u}+\nabla \pi=\boldsymbol{f} \text { and } \operatorname{div} \boldsymbol{u}=h \text { in } \mathbb{R}^{n},
$$


let us recall the fundamental results on which we are based in the sequel. First, for any $k \in \mathbb{Z}$, we introduce the space

$$
\mathcal{S}_{k}=\left\{(\boldsymbol{\lambda}, \mu) \in \mathcal{P}_{k} \times \mathcal{P}_{k-1}^{\Delta} ; \operatorname{div} \boldsymbol{\lambda}=0,-\Delta \boldsymbol{\lambda}+\nabla \mu=\mathbf{0}\right\} .
$$

Theorem 1.2. (See Alliot and Amrouche [1].) Let $\ell \in \mathbb{Z}$ and assume that

$$
n / p^{\prime} \notin\{1, \ldots, \ell\} \quad \text { and } \quad n / p \notin\{1, \ldots,-\ell\} .
$$

For any $(\boldsymbol{f}, h) \in\left(\boldsymbol{W}_{\ell}^{-1, p}\left(\mathbb{R}^{n}\right) \times W_{\ell}^{0, p}\left(\mathbb{R}^{n}\right)\right) \perp \mathcal{S}_{\left[1+\ell-n / p^{\prime}\right]}$, problem $(S)$ admits a solution $(\boldsymbol{u}, \pi) \in \boldsymbol{W}_{\ell}^{1, p}\left(\mathbb{R}^{n}\right) \times W_{\ell}^{0, p}\left(\mathbb{R}^{n}\right)$, unique up to an element of $\mathcal{S}_{[1-\ell-n / p]}$, with the estimate

$$
\begin{aligned}
\inf _{(\boldsymbol{\lambda}, \mu) \in \mathcal{S}_{[1-\ell-n / p]}}\left(\|\boldsymbol{u}+\boldsymbol{\lambda}\|_{\boldsymbol{W}_{\ell}^{1, p}\left(\mathbb{R}^{n}\right)}+\|\pi+\mu\|_{W_{\ell}^{0, p}\left(\mathbb{R}^{n}\right)}\right) & \\
& \leqslant C\left(\|\boldsymbol{f}\|_{\boldsymbol{W}_{\ell}^{-1, p}\left(\mathbb{R}^{n}\right)}+\|h\|_{W_{\ell}^{0, p}\left(\mathbb{R}^{n}\right)}\right) .
\end{aligned}
$$

Theorem 1.3. (See Alliot and Amrouche [1].) Let $\ell \in \mathbb{Z}$ and $m \geqslant 1$ be two integers and assume that

$$
n / p^{\prime} \notin\{1, \ldots, \ell+1\} \quad \text { and } \quad n / p \notin\{1, \ldots,-\ell-m\} .
$$

For any $(\boldsymbol{f}, h) \in\left(\boldsymbol{W}_{m+\ell}^{m-1, p}\left(\mathbb{R}^{n}\right) \times W_{m+\ell}^{m, p}\left(\mathbb{R}^{n}\right)\right) \perp \mathcal{S}_{\left[1+\ell-n / p^{\prime}\right]}$, problem $(S)$ admits a solution $(\boldsymbol{u}, \pi) \in \boldsymbol{W}_{m+\ell}^{m+1, p}\left(\mathbb{R}^{n}\right) \times W_{m+\ell}^{m, p}\left(\mathbb{R}^{n}\right)$, unique up to an element of $\mathcal{S}_{[1-\ell-n / p]}$, with the estimate

$$
\begin{aligned}
& \inf _{(\boldsymbol{\lambda}, \mu) \in \mathcal{S}_{[1-\ell-n / p]}}\left(\|\boldsymbol{u}+\boldsymbol{\lambda}\|_{\boldsymbol{W}_{m+\ell}^{m+1, p}\left(\mathbb{R}^{n}\right)}+\|\pi+\mu\|_{W_{m+\ell}^{m, p}\left(\mathbb{R}^{n}\right)}\right) \\
& \leqslant C\left(\|\boldsymbol{f}\|_{\boldsymbol{W}_{m+\ell}^{m-1, p}\left(\mathbb{R}^{n}\right)}+\|h\|_{W_{m+\ell}^{m, p}\left(\mathbb{R}^{n}\right)}\right) .
\end{aligned}
$$

2. Generalized solutions for the weight zero. In this section, we will concentrate on the central case of weight zero - that is solutions $(\boldsymbol{u}, \pi)$ which belong to $\boldsymbol{W}_{0}^{1, p}\left(\mathbb{R}_{+}^{n}\right) \times L^{p}\left(\mathbb{R}_{+}^{n}\right)$ - This restriction allows us to avoid the questions of kernel and above all of compatibility conditions for the data. However, in the next section, we will rest on this construction to envisage a wide class of weights.

First, we will establish the result about the generalized solutions to $\left(S^{\sharp}\right)$ in the homogeneous case. The method is similar to the one employed for the Dirichlet conditions (see [7]), but the auxiliary problems and the arguments for their resolution are appreciably different.

2.1. The homogeneous case. Here, we assume that $\boldsymbol{f}=\mathbf{0}$ and $h=0$.

Proposition 2.1. For any $g_{n} \in W_{0}^{1-1 / p, p}(\Gamma)$ and $\boldsymbol{g}^{\prime} \in \boldsymbol{W}_{0}^{-1 / p, p}(\Gamma)$ such that $\boldsymbol{g}^{\prime} \perp \mathbb{R}^{n-1}$ if $n \leqslant p^{\prime}$, the Stokes problem

$$
\begin{aligned}
-\Delta \boldsymbol{u}+\nabla \pi & =\mathbf{0} & & \text { in } \mathbb{R}_{+}^{n}, \\
\operatorname{div} \boldsymbol{u} & =0 & & \text { in } \mathbb{R}_{+}^{n}, \\
u_{n} & =g_{n} & & \text { on } \Gamma, \\
\partial_{n} \boldsymbol{u}^{\prime} & =\boldsymbol{g}^{\prime} & & \text { on } \Gamma,
\end{aligned}
$$


has a solution $(\boldsymbol{u}, \pi) \in \boldsymbol{W}_{0}^{1, p}\left(\mathbb{R}_{+}^{n}\right) \times L^{p}\left(\mathbb{R}_{+}^{n}\right)$, unique if $n>p$, unique up to an element of $\mathbb{R}^{n-1} \times\{0\}^{2}$ if $n \leqslant p$, with the estimate

$$
\begin{aligned}
\inf _{\boldsymbol{\xi} \in \mathbb{R}^{n-1} \times\{0\}}\|\boldsymbol{u}+\boldsymbol{\xi}\|_{\boldsymbol{W}_{0}^{1, p}\left(\mathbb{R}_{+}^{n}\right)}+\|\pi\|_{L^{p}\left(\mathbb{R}_{+}^{n}\right)} & \\
& \leqslant C\left(\left\|g_{n}\right\|_{W_{0}^{1-1 / p, p}(\Gamma)}+\left\|\boldsymbol{g}^{\prime}\right\|_{\boldsymbol{W}_{0}^{-1 / p, p}(\Gamma)}\right)
\end{aligned}
$$

if $n \leqslant p$, and the same without $\boldsymbol{\xi}$ if $n>p$.

Remark 2.2. Before giving the proof, let us notice that this problem is not standard. Indeed, we find the velocity field $\boldsymbol{u}$ in $\boldsymbol{W}_{0}^{1, p}\left(\mathbb{R}_{+}^{n}\right)$ with a boundary condition $\partial_{n} \boldsymbol{u}^{\prime}=\boldsymbol{g}^{\prime} \in \boldsymbol{W}_{0}^{-1 / p, p}(\Gamma)$ for its tangential components.

It is possible because $\Delta^{2} \boldsymbol{u}=\mathbf{0}$ in $\mathbb{R}_{+}^{n}$ and then, we find an ad hoc space in which we can give a sense (see [6, Lemma 4.8]) to the trace of $\partial_{n} \boldsymbol{u}^{\prime}$ precisely in the space $\boldsymbol{W}_{0}^{-1 / p, p}(\Gamma)$.

Proof. (i) Firstly, we reduce system (2.1) to three problems on the fundamental operators $\Delta^{2}$ and $\Delta$.

According to (2.1b) and applying the operators div and $\Delta$ to (2.1a), we get both $\Delta \pi=0$ and $\Delta^{2} \boldsymbol{u}=\mathbf{0}$ in $\mathbb{R}_{+}^{n}$.

From the boundary condition (2.1c), we take out

$$
\forall i \in\{1,2, \ldots, n-1\}, \quad \partial_{i}^{2} u_{n}=\partial_{i}^{2} g_{n} \quad \text { on } \Gamma .
$$

In addition, from $(2.1 \mathrm{~d})$, we take out

$$
\partial_{n}^{2} u_{n}=\partial_{n}\left(\partial_{n} u_{n}\right)=\partial_{n}\left(-\operatorname{div}^{\prime} \boldsymbol{u}^{\prime}\right)=-\operatorname{div}^{\prime} \boldsymbol{g}^{\prime} \quad \text { on } \Gamma
$$

hence, the boundary condition

$$
\Delta u_{n}=\Delta^{\prime} g_{n}-\operatorname{div}^{\prime} \boldsymbol{g}^{\prime} \quad \text { on } \Gamma,
$$

where $\Delta^{\prime}=\sum_{j=1}^{n-1} \partial_{j}^{2}$. So, we get the following biharmonic problem

$$
(B): \quad \Delta^{2} u_{n}=0 \text { in } \mathbb{R}_{+}^{n}, \quad u_{n}=g_{n} \text { and } \Delta u_{n}=\Delta^{\prime} g_{n}-\operatorname{div}^{\prime} \boldsymbol{g}^{\prime} \text { on } \Gamma \text {. }
$$

Moreover, we have two Neumann problems

$$
\begin{array}{ll}
(N 1): & \Delta \pi=0 \text { in } \mathbb{R}_{+}^{n}, \quad \partial_{n} \pi=\Delta u_{n} \text { on } \Gamma, \\
(N 2): & \Delta \boldsymbol{u}^{\prime}=\nabla^{\prime} \pi \text { in } \mathbb{R}_{+}^{n}, \quad \partial_{n} \boldsymbol{u}^{\prime}=\boldsymbol{g}^{\prime} \text { on } \Gamma .
\end{array}
$$

(ii) Now, we will solve these three problems.

Step 1: We deal with problem $(B)$. Denoting $z_{n}=\Delta u_{n}$, we can split our problem in the following two Dirichlet problems:

$$
\begin{aligned}
& \Delta z_{n}=0 \text { in } \mathbb{R}_{+}^{n}, \quad z_{n}=\Delta^{\prime} g_{n}-\operatorname{div}^{\prime} \boldsymbol{g}^{\prime} \text { on } \Gamma, \\
& \Delta u_{n}=z_{n} \text { in } \mathbb{R}_{+}^{n}, \quad u_{n}=g_{n} \text { on } \Gamma .
\end{aligned}
$$

Concerning (2.2), we notice that $\Delta^{\prime} g_{n}-\operatorname{div}^{\prime} \boldsymbol{g}^{\prime} \in W_{0}^{-1-1 / p, p}(\Gamma)$, then we can apply the result on the singular boundary conditions for the homogeneous Dirichlet problem (see [7, Theorem 3.5]), provided the following orthogonality condition is satisfied:

$$
\forall \varphi \in \mathcal{A}_{\left[3-n / p^{\prime}\right]}^{\Delta}, \quad\left\langle\Delta^{\prime} g_{n}-\operatorname{div}^{\prime} \boldsymbol{g}^{\prime}, \partial_{n} \varphi\right\rangle_{W_{0}^{-1-1 / p, p}(\Gamma) \times W_{0}^{2-1 / p^{\prime}, p^{\prime}}(\Gamma)}=0 .
$$


According to the degre of polynomials in $\mathcal{A}_{\left[3-n / p^{\prime}\right]}^{\Delta}$, this condition reduces to $\boldsymbol{g}^{\prime} \perp$ $\mathcal{P}_{\left[1-n / p^{\prime}\right]}$, which is precisely the assumption of Proposition 2.1. Thus problem (2.2) has a unique solution $z_{n} \in W_{0}^{-1, p}\left(\mathbb{R}_{+}^{n}\right)$.

Concerning (2.3), we can apply the result on the generalized solutions to the Dirichlet problem (see $\left[3\right.$, Theorem 3.1]) without any condition since $\mathcal{A}_{\left[1-n / p^{\prime}\right]}^{\Delta}=\{0\}$. Thus problem (2.3) has a unique solution $u_{n} \in W_{0}^{1, p}\left(\mathbb{R}_{+}^{n}\right)$.

Step 2: Next, we study problem $(N 1)$. Since $\Delta u_{n} \in W_{0}^{-1, p}\left(\mathbb{R}_{+}^{n}\right)$, it is necessary to check that the trace of $\Delta u_{n}$ has meaning. We have both $\Delta u_{n} \in W_{0}^{-1, p}\left(\mathbb{R}_{+}^{n}\right)$ and $\Delta^{2} u_{n}=0$, then it follows that $\Delta u_{n} \in W_{0}^{-1-1 / p, p}(\Gamma)$ (see [7, Lemma 3.7]). Next, the result on the singular boundary conditions for the homogeneous Neumann problem (see [4] or [7, Theorem 3.3]) holds, provided the following orthogonality condition is satisfied:

$$
\forall \varphi \in \mathcal{N}_{\left[2-n / p^{\prime}\right]}^{\Delta}, \quad\left\langle\Delta u_{n}, \varphi\right\rangle_{W_{0}^{-1-1 / p, p}(\Gamma) \times W_{0}^{2-1 / p^{\prime}, p^{\prime}}(\Gamma)}=0 .
$$

But, according to the degre of polynomials in $\mathcal{N}_{\left[2-n / p^{\prime}\right]}^{\Delta}$, it is clear that this condition is always satisfied. It implies the existence of a unique solution $\pi \in L^{p}\left(\mathbb{R}_{+}^{n}\right)$ to problem $(N 1)$.

Step 3: Finally, we are dealing with problem (N2). We split it in two parts:

$$
\Delta \boldsymbol{v}^{\prime}=\nabla^{\prime} \pi \text { in } \mathbb{R}_{+}^{n}, \quad \partial_{n} \boldsymbol{v}^{\prime}=\mathbf{0} \text { on } \Gamma,
$$

and

$$
\Delta \boldsymbol{z}^{\prime}=\mathbf{0} \text { in } \mathbb{R}_{+}^{n}, \quad \partial_{n} \boldsymbol{z}^{\prime}=\boldsymbol{g}^{\prime} \text { on } \Gamma .
$$

To solve (2.4), we introduce the auxiliary problem

$$
\Delta w=\pi \text { in } \mathbb{R}_{+}^{n}, \quad \partial_{n} w=0 \text { on } \Gamma .
$$

Since we have $\pi \in L^{p}\left(\mathbb{R}_{+}^{n}\right)$, problem (2.6) has a solution $w \in W_{0}^{2, p}\left(\mathbb{R}_{+}^{n}\right)$, unique up to an element of $\mathcal{N}_{[2-n / p]}^{\Delta}$ (see [4]). Next, it suffices to put $\boldsymbol{v}^{\prime}=\nabla^{\prime} w$ to obtain a solution (non-unique) $\boldsymbol{v}^{\prime} \in \boldsymbol{W}_{0}^{1, p}\left(\mathbb{R}_{+}^{n}\right)$ to problem (2.4).

For problem (2.5), with $\boldsymbol{g}^{\prime} \in \boldsymbol{W}_{0}^{-1 / p, p}(\Gamma)$, we must use an intermediate result for the Neumann problem (see [7, Theorem 3.4]). The compatibility condition is written in this case: $\boldsymbol{g}^{\prime} \perp \mathcal{P}_{\left[1-n / p^{\prime}\right]}$. Thus it is realized by the assumption of Proposition 2.1. So, this problem has a solution $\boldsymbol{z}^{\prime} \in \boldsymbol{W}_{0}^{1, p}\left(\mathbb{R}_{+}^{n}\right)$, unique up to an element of $\mathcal{P}_{[1-n / p]}$. (N2).

Then, it is clear that the function $\boldsymbol{u}^{\prime}=\boldsymbol{v}^{\prime}+\boldsymbol{z}^{\prime} \in \boldsymbol{W}_{0}^{1, p}\left(\mathbb{R}_{+}^{n}\right)$ is solution to problem

(iii) Conversely, it is necessary to show that from $u_{n}, \pi, \boldsymbol{u}^{\prime}$, we get a solution $(\boldsymbol{u}, \pi)$ of the original problem $(2.1)$.

From previous it is clear that

$$
\begin{aligned}
-\Delta \boldsymbol{u}^{\prime}+\nabla^{\prime} \pi & =0 & & \text { in } \mathbb{R}_{+}^{n} \\
u_{n} & =g_{n} & & \text { on } \Gamma, \\
\partial_{n} \boldsymbol{u}^{\prime} & =\boldsymbol{g}^{\prime} & & \text { on } \Gamma .
\end{aligned}
$$

It remains to prove that

$$
-\Delta u_{n}+\partial_{n} \pi=0 \text { in } \mathbb{R}_{+}^{n}
$$


and finally, the relation $(2.1 \mathrm{~b})$.

For (2.7), thanks to the first equations of $(B)$ and $(N 1)$, we get

$$
\Delta\left(\Delta u_{n}-\partial_{n} \pi\right)=\Delta^{2} u_{n}=0 \quad \text { in } \mathbb{R}_{+}^{n} .
$$

With the boundary condition of $(N 1)$, it follows that $\Delta u_{n}-\partial_{n} \pi$ satisfies the problem

$$
\Delta\left(\Delta u_{n}-\partial_{n} \pi\right)=0 \text { in } \mathbb{R}_{+}^{n}, \quad \Delta u_{n}-\partial_{n} \pi=0 \text { on } \Gamma .
$$

As well $\Delta u_{n}-\partial_{n} \pi \in W_{0}^{-1, p}\left(\mathbb{R}_{+}^{n}\right)$, then by a uniqueness argument, we necessarily have $\Delta u_{n}-\partial_{n} \pi=0$ in $\mathbb{R}_{+}^{n}$ (see [7, Theorem 3.5]).

For (2.1b), the boundary conditions of (N2) imply $\partial_{n} \operatorname{div}^{\prime} \boldsymbol{u}^{\prime}=\operatorname{div}^{\prime} \boldsymbol{g}^{\prime}$ on $\Gamma$. Besides, from the boundary conditions of $(B)$, we get $\partial_{n}^{2} u_{n}=-\operatorname{div}^{\prime} \boldsymbol{g}^{\prime}$ on $\Gamma$. Then we have

$$
\partial_{n} \operatorname{div} \boldsymbol{u}=\partial_{n} \operatorname{div}^{\prime} \boldsymbol{u}^{\prime}+\partial_{n}^{2} u_{n}=\operatorname{div}^{\prime} \boldsymbol{g}^{\prime}-\operatorname{div}^{\prime} \boldsymbol{g}^{\prime}=0 \quad \text { on } \Gamma .
$$

So, $\operatorname{div} \boldsymbol{u}$ satisfies the problem

$$
\Delta \operatorname{div} \boldsymbol{u}=0 \text { in } \mathbb{R}_{+}^{n}, \quad \partial_{n} \operatorname{div} \boldsymbol{u}=0 \quad \text { on } \Gamma .
$$

As well $\operatorname{div} \boldsymbol{u} \in L^{p}\left(\mathbb{R}_{+}^{n}\right)$, hence $\operatorname{div} \boldsymbol{u}=0$ in $\mathbb{R}_{+}^{n}$ (see [4] or [7, Theorem 3.3]).

(iv) Concerning the uniqueness question, we have notice that $u_{n}$ and $\pi$ are unique. Let $\boldsymbol{u}^{\prime}=\left(u_{i}\right)_{1 \leqslant i \leqslant n-1}$ and $\boldsymbol{u}_{\dagger}^{\prime}=\left(u_{i}^{\dagger}\right)_{1 \leqslant i \leqslant n-1}$ be solutions to $(N 2)$, then

$$
\begin{array}{rll}
\Delta\left(u_{i}-u_{i}^{\dagger}\right) & =0 & \text { in } \mathbb{R}_{+}^{n}, \\
\partial_{n}\left(u_{i}-u_{i}^{\dagger}\right) & =0 & \text { on } \Gamma,
\end{array}
$$

where $u_{i}-u_{i}^{\dagger} \in W_{0}^{1, p}\left(\mathbb{R}_{+}^{n}\right)$. So we can deduce that $u_{i}-u_{i}^{\dagger} \in \mathcal{N}_{[1-n / p]}^{\Delta}$ (see [4]). It remains to remark that $\mathcal{N}_{[1-n / p]}^{\Delta}=\mathbb{R}$ if $n \leqslant p$, and $\mathcal{N}_{[1-n / p]}^{\Delta}=\{0\}$ if $n>p$.

Finally, the estimate of Proposition 2.1 is a straightforward consequence of the Banach Theorem. Let us notice that we also can get it from the estimates of the auxiliary problems as we showed in [7] for the no-slip boundary conditions.

2.2. The nonhomogeneous case. Resting on the previous result, we now can deal with the complete problem.

THEOREM 2.3. Assume that $\frac{n}{p^{\prime}} \neq 1$. For any $\boldsymbol{f} \in \boldsymbol{W}_{1}^{0, p}\left(\mathbb{R}_{+}^{n}\right), h \in W_{1}^{1, p}\left(\mathbb{R}_{+}^{n}\right)$, $g_{n} \in W_{0}^{1-1 / p, p}(\Gamma)$ and $\boldsymbol{g}^{\prime} \in \boldsymbol{W}_{0}^{-1 / p, p}(\Gamma)$, satisfying the following compatibility condition if $n<p^{\prime}$ :

$$
\forall i \in\{1, \ldots, n-1\}, \quad \int_{\mathbb{R}_{+}^{n}} f_{i} \mathrm{~d} x=\left\langle g_{i}, 1\right\rangle_{W_{0}^{-1 / p, p}(\Gamma) \times W_{0}^{1 / p, p^{\prime}}(\Gamma)},
$$

problem $\left(S^{\sharp}\right)$ admits a solution $(\boldsymbol{u}, \pi) \in \boldsymbol{W}_{0}^{1, p}\left(\mathbb{R}_{+}^{n}\right) \times L^{p}\left(\mathbb{R}_{+}^{n}\right)$, unique if $n>p$, unique up to an element of $\mathbb{R}^{n-1} \times\{0\}^{2}$ if $n \leqslant p$, with the estimate

$$
\begin{aligned}
& \inf _{\boldsymbol{\xi} \in \mathbb{R}^{n-1} \times\{0\}}\|\boldsymbol{u}+\boldsymbol{\xi}\|_{\boldsymbol{W}_{0}^{1, p}\left(\mathbb{R}_{+}^{n}\right)}+\|\pi\|_{L^{p}\left(\mathbb{R}_{+}^{n}\right)} \\
& \quad \leqslant C\left(\|\boldsymbol{f}\|_{\boldsymbol{W}_{1}^{0, p}\left(\mathbb{R}_{+}^{n}\right)}+\|h\|_{W_{1}^{1, p}\left(\mathbb{R}_{+}^{n}\right)}+\left\|g_{n}\right\|_{W_{0}^{1-1 / p, p}(\Gamma)}+\left\|\boldsymbol{g}^{\prime}\right\|_{\boldsymbol{W}_{0}^{-1 / p, p}(\Gamma)}\right)
\end{aligned}
$$

if $n \leqslant p$, and the same without $\boldsymbol{\xi}$ if $n>p$. 
Proof. We can give a proof quite similar to the one of the nonhomogeneous case for the Stokes system with Dirichlet boundary conditions, by extension of the data $f$ and $h$ to the whole space (see [7]). But another way is to combine this result with the homogeneous case for the Stokes system with Navier boundary conditions. We will follow this one.

Firstly, we introduce the auxiliary problem

$$
\begin{aligned}
& -\Delta \boldsymbol{z}+\nabla \eta=\boldsymbol{f} \text { in } \mathbb{R}_{+}^{n}, \\
& \operatorname{div} \boldsymbol{z}=h \quad \text { in } \mathbb{R}_{+}^{n}, \\
& \boldsymbol{z}=\mathbf{0} \text { on } \Gamma \text {. }
\end{aligned}
$$

With the assumption $\frac{n}{p^{\prime}} \neq 1$, we know that problem (2.11) admits a unique solution $(\boldsymbol{z}, \eta) \in \boldsymbol{W}_{1}^{2, p}\left(\mathbb{R}_{+}^{n}\right) \times W_{1}^{1, p}\left(\mathbb{R}_{+}^{n}\right)$ (see [7, Theorem 5.2]). Thus we can deduce that $\left.\partial_{n} \boldsymbol{z}^{\prime}\right|_{\Gamma} \in \boldsymbol{W}_{1}^{1-1 / p, p}(\Gamma)$. In addition, we can notice that we have the imbeddings $W_{1}^{2, p}\left(\mathbb{R}_{+}^{n}\right) \hookrightarrow W_{0}^{1, p}\left(\mathbb{R}_{+}^{n}\right)$ and $W_{1}^{1, p}\left(\mathbb{R}_{+}^{n}\right) \hookrightarrow L^{p}\left(\mathbb{R}_{+}^{n}\right)$ without condition, whereas we have $W_{1}^{1-1 / p, p}(\Gamma) \hookrightarrow W_{0}^{-1 / p, p}(\Gamma)$ only if $\frac{n}{p^{\prime}} \neq 1$.

Indeed, we can break it down into

$$
W_{1}^{1-1 / p, p}(\Gamma) \hookrightarrow W_{1 / p}^{0, p}(\Gamma) \quad \text { and } \quad W_{1 / p}^{0, p}(\Gamma) \hookrightarrow W_{0}^{-1 / p, p}(\Gamma) .
$$

The first imbedding holds without condition. By duality, the second one is equivalent to $W_{0}^{1 / p, p^{\prime}}(\Gamma) \hookrightarrow W_{-1 / p}^{0, p^{\prime}}(\Gamma)$, which holds if $\frac{n-1}{p^{\prime}} \neq \frac{1}{p}$, i.e. $\frac{n}{p^{\prime}} \neq 1$.

So, $(\boldsymbol{z}, \eta) \in \boldsymbol{W}_{0}^{1, p}\left(\mathbb{R}_{+}^{n}\right) \times L^{p}\left(\mathbb{R}_{+}^{n}\right)$ and above all $\gamma_{1} \boldsymbol{z}^{\prime} \in \boldsymbol{W}_{0}^{-1 / p, p}(\Gamma)$, which allows us to consider the second auxiliary problem

$$
\begin{array}{rlll}
-\Delta \boldsymbol{v}+\nabla \vartheta=\mathbf{0} & \text { and } & \operatorname{div} \boldsymbol{v}=0 & \text { in } \mathbb{R}_{+}^{n}, \\
v_{n}=g_{n} & \text { and } & \partial_{n} \boldsymbol{v}^{\prime}=\boldsymbol{g}^{\prime}-\partial_{n} \boldsymbol{z}^{\prime} & \text { on } \Gamma,
\end{array}
$$

where $\boldsymbol{g}^{\prime}-\left.\partial_{n} \boldsymbol{z}^{\prime}\right|_{\Gamma}=\boldsymbol{g}^{\prime}-\gamma_{1} \boldsymbol{z}^{\prime} \in \boldsymbol{W}_{0}^{-1 / p, p}(\Gamma)$. Then, Proposition 2.1 yields $(\boldsymbol{v}, \vartheta) \in$ $\boldsymbol{W}_{0}^{1, p}\left(\mathbb{R}_{+}^{n}\right) \times L^{p}\left(\mathbb{R}_{+}^{n}\right)$ solution to $(2.12)$, provided the orthogonality condition

$$
\forall \boldsymbol{\varphi}^{\prime} \in \mathbb{R}^{n-1}, \quad\left\langle\boldsymbol{g}^{\prime}-\gamma_{1} \boldsymbol{z}^{\prime}, \boldsymbol{\varphi}^{\prime}\right\rangle_{\boldsymbol{W}_{0}^{-1 / p, p}(\Gamma) \times \boldsymbol{W}_{0}^{1 / p, p^{\prime}}(\Gamma)}=0
$$

is satisfied if $n<p^{\prime}$. Now, we must write this condition by only means of data. It suffices to notice that we have for all $\varphi \in \mathbb{R}^{n-1} \times\{0\}$,

$$
\begin{aligned}
\int_{\mathbb{R}_{+}^{n}} \boldsymbol{f} \cdot \boldsymbol{\varphi} \mathrm{d} x & =\int_{\mathbb{R}_{+}^{n}}(-\Delta \boldsymbol{z}+\nabla \eta) \cdot \boldsymbol{\varphi} \mathrm{d} x \\
& =\left\langle\gamma_{1} \boldsymbol{z}^{\prime}, \boldsymbol{\varphi}^{\prime}\right\rangle_{\boldsymbol{W}_{0}^{-1 / p, p}(\Gamma) \times \boldsymbol{W}_{0}^{1 / p, p^{\prime}}(\Gamma)},
\end{aligned}
$$

to deduce that the condition (2.13) is written

$$
\forall \boldsymbol{\varphi}^{\prime} \in \mathbb{R}^{n-1}, \quad \int_{\mathbb{R}_{+}^{n}} \boldsymbol{f}^{\prime} \cdot \boldsymbol{\varphi}^{\prime} \mathrm{d} x=\left\langle\boldsymbol{g}^{\prime}, \boldsymbol{\varphi}^{\prime}\right\rangle_{\boldsymbol{W}_{0}^{-1 / p, p}(\Gamma) \times \boldsymbol{W}_{0}^{1 / p, p^{\prime}}(\Gamma)},
$$

that is, more simply, the compatibility condition (2.10).

Then, the pair $(\boldsymbol{u}, \pi)=(\boldsymbol{v}+\boldsymbol{z}, \vartheta+\eta)$ which belongs to $\boldsymbol{W}_{0}^{1, p}\left(\mathbb{R}_{+}^{n}\right) \times L^{p}\left(\mathbb{R}_{+}^{n}\right)$ is a solution to $\left(S^{\sharp}\right)$.

Finally, the uniqueness of solutions to $\left(S^{\sharp}\right)$ is a straightforward consequence of Proposition 2.1. 
Remark 2.4. Unlike Dirichlet boundary conditions, with Navier conditions it is not reasonable to consider data $(\boldsymbol{f}, h)$ in $\boldsymbol{W}_{0}^{-1, p}\left(\mathbb{R}_{+}^{n}\right) \times L^{p}\left(\mathbb{R}_{+}^{n}\right)$. Indeed, with such data for problem (2.11) we should get the velocity field $\boldsymbol{z}$ in the space $W_{0}^{1, p}\left(\mathbb{R}_{+}^{n}\right)$ and we cannot give a sense to the trace of $\partial_{n} z^{\prime}$ in that case without ad hoc assumption. This limitation is not due to the method employed here, it is the same situation as in the Neumann problem for the Laplacian (see [4]).

3. A wide class of behaviour at infinity. Naturally, this question will be solved by the consideration of a scale of weights which extends the weight zero of the previous section. After the study of the kernel of the operator associated to this problem, we will show that the method established for the homogeneous system with the weight zero works in fact for any weight. The main difficulty is to get the compatibility conditions in all the auxiliary problems from the one of the original problem. Next, the treatment of the nonhomogeneous system will be noticeably different.

3.1. The kernel. In the half-space, a key for this question is the reflection principle. We can find an extensive study of this principle in the work of Farwig (see [14]). With these boundary conditions, the reflection principle is simpler than the one for the Dirichlet conditions and it can be deduced from the classical Schwarz reflection principle for the harmonic functions.

Let $\ell \in \mathbb{Z}$ and $(\boldsymbol{u}, \pi) \in \boldsymbol{W}_{\ell}^{1, p}\left(\mathbb{R}_{+}^{n}\right) \times W_{\ell}^{0, p}\left(\mathbb{R}_{+}^{n}\right)$ be an element of the kernel of the Stokes operator with Navier boundary conditions - that is a solution of (2.1) with homogeneous boundary conditions - , then the unique extention $(\tilde{\boldsymbol{u}}, \tilde{\pi})$ of $(\boldsymbol{u}, \pi)$ to the whole space, satisfying

$$
-\Delta \tilde{\boldsymbol{u}}+\nabla \tilde{\pi}=\mathbf{0} \text { and } \operatorname{div} \tilde{\boldsymbol{u}}=0 \quad \text { in } \mathbb{R}^{n},
$$

is given by the continuation formulae: for all $x=\left(x^{\prime}, x_{n}\right) \in \mathbb{R}_{-}^{n}$,

$$
\tilde{\boldsymbol{u}}^{\prime}(x)=\boldsymbol{u}^{\prime}\left(x^{*}\right), \tilde{u}_{n}(x)=-u_{n}\left(x^{*}\right), \tilde{\pi}(x)=\pi\left(x^{*}\right), \quad \text { where } x^{*}=\left(x^{\prime},-x_{n}\right) .
$$

Moreover, such $\tilde{\pi}$ and $\tilde{\boldsymbol{u}}$ are respectively harmonic and biharmonic tempered distributions in $\mathbb{R}^{n}$, thus polynomials. For all $k \in \mathbb{Z}$, let us denote

$$
\begin{aligned}
\mathcal{S}_{k}^{\sharp}=\left\{(\boldsymbol{\lambda}, \mu) \in \mathcal{P}_{k}^{\Delta^{2}} \times \mathcal{P}_{k-1}^{\Delta} ;-\Delta \boldsymbol{\lambda}+\nabla \mu=\mathbf{0} \text { and } \operatorname{div} \boldsymbol{\lambda}\right. & =0 \text { in } \mathbb{R}_{+}^{n}, \\
\partial_{n} \boldsymbol{\lambda}^{\prime} & \left.=\mathbf{0} \text { and } \lambda_{n}=0 \text { on } \Gamma\right\} .
\end{aligned}
$$

According to the maximum degre of polynomials in weighted Sobolev spaces (see [2]), we can characterize this kernel as follows:

Corollary 3.1. Let $\ell \in \mathbb{Z}$ with hypothesis (1.6), then the kernel of the Stokes operator with Navier boundary conditions in $\boldsymbol{W}_{\ell}^{1, p}\left(\mathbb{R}_{+}^{n}\right) \times W_{\ell}^{0, p}\left(\mathbb{R}_{+}^{n}\right)$ is the space $\mathcal{S}_{[1-\ell-n / p]}^{\sharp} \cdot$

In fact this kernel does not depend on the regularity according to the Sobolev imbbedings. More precisely, we have the following result:

Corollary 3.2. Let $\ell \in \mathbb{Z}$ and $m \in \mathbb{N}^{*}$ with (1.7), then the kernel of the Stokes operator with Navier boundary conditions in $\boldsymbol{W}_{m+\ell}^{m+1, p}\left(\mathbb{R}_{+}^{n}\right) \times W_{m+\ell}^{m, p}\left(\mathbb{R}_{+}^{n}\right)$ is the space $\mathcal{S}_{[1-\ell-n / p]}^{\sharp}$.

Using an idea due to T. Z. Boulmezaoud (see [11]), we also can express this space from the polynomials spaces $\mathcal{A}_{k}^{\Delta}$ and $\mathcal{N}_{k}^{\Delta}$ which define the kernels of the Laplacian with Dirichlet and Neumann boundary conditions in the half-space. With this aim, we 
will use the operator $\Pi_{N}$ - introduced in [5] for the biharmonic problem - defined as follows:

$$
\forall s \in \mathcal{N}_{k}^{\Delta}, \quad \Pi_{N} s\left(x^{\prime}, x_{n}\right)=\frac{1}{2} x_{n} \int_{0}^{x_{n}} s\left(x^{\prime}, t\right) \mathrm{d} t
$$

and satisfying for all $s \in \mathcal{N}_{k}^{\Delta}, \Delta \Pi_{N} s=s$ in $\mathbb{R}_{+}^{n}$ and $\Pi_{N} s=\partial_{n} \Pi_{N} s=0$ on $\Gamma$.

Proposition 3.3. Let $\ell \in \mathbb{Z}$. The pair $(\boldsymbol{\lambda}, \mu) \in \mathcal{S}_{[1-\ell-n / p]}^{\sharp}$ if and only if there exists $\varphi \in \mathcal{N}_{[1-\ell-n / p]}^{\Delta} \times \mathcal{A}_{[1-\ell-n / p]}^{\Delta}$ such that

$$
\boldsymbol{\lambda}=\boldsymbol{\varphi}-\nabla \Pi_{N} \operatorname{div} \boldsymbol{\varphi}, \quad \mu=-\operatorname{div} \boldsymbol{\varphi}
$$

Proof. Given $(\boldsymbol{\lambda}, \mu) \in \mathcal{S}_{[1-\ell-n / p]}^{\sharp}$, we have $\Delta \mu=0$ in $\mathbb{R}_{+}^{n}$ and $\partial_{n} \mu=0$ on $\Gamma$, hence $\mu \in \mathcal{N}_{[1-\ell-n / p]}^{\Delta}$. So we can write $\Delta\left(\boldsymbol{\lambda}-\nabla \Pi_{N} \mu\right)=\Delta \boldsymbol{\lambda}-\nabla \mu=\mathbf{0}$, which implies the existence of $\varphi \in \mathcal{P}_{[1-\ell-n / p]}^{\Delta}$ such that

$$
\varphi=\lambda-\nabla \Pi_{N} \mu \text {. }
$$

In fact, we can see that $\boldsymbol{\varphi} \in \mathcal{N}_{[1-\ell-n / p]}^{\Delta} \times \mathcal{A}_{[1-\ell-n / p]}^{\Delta}$ by considerations on the parity of $\lambda^{\prime}, \lambda_{n}$ and $\nabla \Pi_{N} \mu$. In addition, applying the operator div to (3.2), we get $\operatorname{div} \varphi=-\mu$, which yieds (3.1) by substitution in (3.2).

Conversely, we can verify that such a pair $(\boldsymbol{\lambda}, \mu)$ belongs to $\mathcal{S}_{[1-\ell-n / p]}^{\sharp}$.

Remark 3.4. It is clear that if $\ell>0$, this kernel is reduced to $\{\mathbf{0}\}$ and if $\ell=0$, we find $\mathbb{R}^{n-1} \times\{0\}^{2}$ as in Proposition 2.1 and Theorem 2.3. Symmetrically, if $\ell>0$, it appears a compatibility condition for the data, which extends the one of the weight zero.

3.2. Generalized solutions. Here is the generalization of Theorem 2.3 for any weight $\ell \in \mathbb{Z}$. This result will be completely proved at the end of this section.

THEOREM 3.5. Let $\ell \in \mathbb{Z}$ and assume that

$$
n / p^{\prime} \notin\{1, \ldots, \ell+1\} \quad \text { and } \quad n / p \notin\{1, \ldots,-\ell\} .
$$

For any $\boldsymbol{f} \in \boldsymbol{W}_{\ell+1}^{0, p}\left(\mathbb{R}_{+}^{n}\right), h \in W_{\ell+1}^{1, p}\left(\mathbb{R}_{+}^{n}\right), g_{n} \in W_{\ell}^{1-1 / p, p}(\Gamma), \boldsymbol{g}^{\prime} \in \boldsymbol{W}_{\ell}^{-1 / p, p}(\Gamma)$, satisfying the compatibility condition

$$
\begin{aligned}
& \forall \boldsymbol{\varphi} \in \mathcal{N}_{\left[1+\ell-n / p^{\prime}\right]}^{\Delta} \times \mathcal{A}_{\left[1+\ell-n / p^{\prime}\right]}^{\Delta}, \\
& \int_{\mathbb{R}_{+}^{n}}(\boldsymbol{f}-\nabla h) \cdot \boldsymbol{\varphi} \mathrm{d} x+\left\langle\operatorname{div} \boldsymbol{f}, \Pi_{N} \operatorname{div} \boldsymbol{\varphi}\right\rangle_{W_{\ell+1}^{-1, p}\left(\mathbb{R}_{+}^{n}\right) \times \stackrel{\circ}{W}_{-\ell-1}^{1, p^{\prime}}\left(\mathbb{R}_{+}^{n}\right)} \\
& \quad+\int_{\Gamma} g_{n} \partial_{n} \varphi_{n} \mathrm{~d} x^{\prime}-\left\langle\boldsymbol{g}^{\prime}, \boldsymbol{\varphi}^{\prime}\right\rangle_{\boldsymbol{W}_{\ell}^{-1 / p, p}(\Gamma) \times \boldsymbol{W}_{-\ell}^{1-1 / p^{\prime}, p^{\prime}}(\Gamma)}=0
\end{aligned}
$$

problem $\left(S^{\sharp}\right)$ admits a solution $(\boldsymbol{u}, \pi) \in \boldsymbol{W}_{\ell}^{1, p}\left(\mathbb{R}_{+}^{n}\right) \times W_{\ell}^{0, p}\left(\mathbb{R}_{+}^{n}\right)$, unique up to an element of $\mathcal{S}_{[1-\ell-n / p]}^{\sharp}$, with the estimate

$$
\begin{aligned}
& \inf _{(\boldsymbol{\lambda}, \mu) \in \mathcal{S}_{[1-\ell-n / p]}^{\sharp}}\left(\|\boldsymbol{u}+\boldsymbol{\lambda}\|_{\boldsymbol{W}_{\ell}^{1, p}\left(\mathbb{R}_{+}^{n}\right)}+\|\pi+\mu\|_{W_{\ell}^{0, p}\left(\mathbb{R}_{+}^{n}\right)}\right) \leqslant \\
& C\left(\|\boldsymbol{f}\|_{\boldsymbol{W}_{\ell+1}^{0, p}\left(\mathbb{R}_{+}^{n}\right)}+\|h\|_{W_{\ell+1}^{0, p}\left(\mathbb{R}_{+}^{n}\right)}+\left\|g_{n}\right\|_{W_{\ell}^{1-1 / p, p}(\Gamma)}+\left\|\boldsymbol{g}^{\prime}\right\|_{\boldsymbol{W}_{\ell}^{-1 / p, p}(\Gamma)}\right) .
\end{aligned}
$$


Since the kernel has been characterized before, now it remains to show the necessity of condition (3.4) and above all the existence of a solution, that is the surjectivity of this operator. As for the weight zero, we will start with the homogeneous problem, then we will consider more regular data on the boundary, to finish by this theorem.

3.3. The compatibility condition. If $(\boldsymbol{u}, \pi)$ is a solution to $\left(S^{\sharp}\right)$, then we have the following Green formula:

$$
\begin{aligned}
\forall(\boldsymbol{\lambda}, \mu) \in \mathcal{S}_{\left[1+\ell-n / p^{\prime}\right]}^{\sharp}, & \int_{\mathbb{R}_{+}^{n}}(-\Delta \boldsymbol{u}+\nabla \pi) \cdot \boldsymbol{\lambda} \mathrm{d} x-\int_{\mathbb{R}_{+}^{n}}(\operatorname{div} \boldsymbol{u}) \mu \mathrm{d} x= \\
& -\int_{\Gamma} u_{n} \partial_{n} \lambda_{n} \mathrm{~d} x^{\prime}+\left\langle\partial_{n} \boldsymbol{u}^{\prime}, \boldsymbol{\lambda}^{\prime}\right\rangle_{\boldsymbol{W}_{\ell}^{-1 / p, p}(\Gamma) \times \boldsymbol{W}_{-\ell}^{1-1 / p^{\prime}, p^{\prime}(\Gamma)}}+\int_{\Gamma} u_{n} \mu \mathrm{d} x^{\prime} .
\end{aligned}
$$

Hence a first formulation of the compatibility condition for data $\boldsymbol{f}, h, g_{n}, \boldsymbol{g}^{\prime}$ :

$$
\begin{aligned}
& \forall(\boldsymbol{\lambda}, \mu) \in \mathcal{S}_{\left[1+\ell-n / p^{\prime}\right]}^{\sharp}, \quad \int_{\mathbb{R}_{+}^{n}} \boldsymbol{f} \cdot \boldsymbol{\lambda} \mathrm{d} x-\int_{\mathbb{R}_{+}^{n}} h \mu \mathrm{d} x= \\
& -\int_{\Gamma} g_{n}\left(\partial_{n} \lambda_{n}-\mu\right) \mathrm{d} x^{\prime}+\left\langle\boldsymbol{g}^{\prime}, \boldsymbol{\lambda}^{\prime}\right\rangle_{\boldsymbol{W}_{\ell}^{-1 / p, p}(\Gamma) \times \boldsymbol{W}_{-\ell}^{1-1 / p^{\prime}, p^{\prime}}(\Gamma)} .
\end{aligned}
$$

Now, in order to use Proposition 3.3, we can observe that

$$
\begin{aligned}
\forall \boldsymbol{\varphi} \in \mathcal{N}_{\left[1+\ell-n / p^{\prime}\right]}^{\Delta} \times \mathcal{A}_{\left[1+\ell-n / p^{\prime}\right]}^{\Delta}, & \\
& \int_{\mathbb{R}_{+}^{n}} \boldsymbol{f} \cdot\left(\nabla \Pi_{N} \operatorname{div} \boldsymbol{\varphi}\right) \mathrm{d} x=\left\langle-\operatorname{div} \boldsymbol{f}, \Pi_{N} \operatorname{div} \boldsymbol{\varphi}\right\rangle_{W_{\ell+1}^{-1, p}\left(\mathbb{R}_{+}^{n}\right) \times \stackrel{\circ}{W_{-\ell-1}^{1, p^{\prime}}\left(\mathbb{R}_{+}^{n}\right)}}
\end{aligned}
$$

and

$$
\int_{\mathbb{R}_{+}^{n}} h \operatorname{div} \varphi \mathrm{d} x=-\int_{\mathbb{R}_{+}^{n}} \nabla h \cdot \varphi \mathrm{d} x .
$$

On the other hand, for the trace terms, we have

$$
\lambda^{\prime}=\varphi^{\prime} \text { and } \partial_{n} \lambda_{n}-\mu=\partial_{n} \varphi_{n} \text { on } \Gamma .
$$

According to Proposition 3.3 and introducing these identities in (3.5), we get (3.4) as second formulation for the compatibility condition.

3.4. Weak and strong solutions in the homogeneous case. Here again, we start with the homogeneous Stokes system (2.1). In fact the method of Subsection 2.1 works for any weight. The extra trouble comes from the compatibility conditions for the auxiliary problems. Following step by step the proof of Proposition 2.1, we throw light on this question.

Proof of Proposition 2.1 revisited. Point (i) is unchanged.

(ii) The compatibility condition (3.4) adapted to problem (2.1) is written

$$
\begin{aligned}
\forall \boldsymbol{\varphi} \in \mathcal{N}_{\left[1+\ell-n / p^{\prime}\right]}^{\Delta} \times \mathcal{A}_{\left[1+\ell-n / p^{\prime}\right]}^{\Delta}, \\
\qquad \int_{\Gamma} g_{n} \partial_{n} \varphi_{n} \mathrm{~d} x^{\prime}-\left\langle\boldsymbol{g}^{\prime}, \boldsymbol{\varphi}^{\prime}\right\rangle_{\boldsymbol{W}_{\ell}^{-1 / p, p}(\Gamma) \times \boldsymbol{W}_{-\ell}^{1-1 / p^{\prime}, p^{\prime}}(\Gamma)}=0,
\end{aligned}
$$


Step 1: Problem (B). For (2.2), the compatibility condition is

$$
\forall \psi \in \mathcal{A}_{\left[3+\ell-n / p^{\prime}\right]}^{\Delta},\left\langle\Delta^{\prime} g_{n}-\operatorname{div}^{\prime} \boldsymbol{g}^{\prime}, \partial_{n} \psi\right\rangle_{\boldsymbol{W}_{\ell}^{-1-1 / p, p}(\Gamma) \times \boldsymbol{W}_{-\ell}^{2-1 / p^{\prime}, p^{\prime}}(\Gamma)}=0
$$

(see [7, Theorem 3.5]). By means of Green formulae, we can rewrite it

$$
\forall \psi \in \mathcal{A}_{\left[3+\ell-n / p^{\prime}\right]}^{\Delta}, \quad \int_{\Gamma} g_{n} \partial_{n} \Delta^{\prime} \psi \mathrm{d} x^{\prime}+\left\langle\boldsymbol{g}^{\prime}, \partial_{n} \nabla^{\prime} \psi\right\rangle_{\boldsymbol{W}_{\ell}^{-1 / p, p}(\Gamma) \times \boldsymbol{W}_{-\ell}^{1-1 / p^{\prime}, p^{\prime}}(\Gamma)}=0 .
$$

Now, to see that it is a consequence of (3.6), it suffices to remark that

$$
\forall \psi \in \mathcal{A}_{\left[3+\ell-n / p^{\prime}\right]}^{\Delta}, \quad \Delta^{\prime} \psi \in \mathcal{A}_{\left[1+\ell-n / p^{\prime}\right]}^{\Delta} \text { and } \partial_{n} \nabla^{\prime} \psi \in \mathcal{N}_{\left[1+\ell-n / p^{\prime}\right]}^{\Delta} .
$$

So, we get $z_{n} \in W_{\ell}^{-1, p}\left(\mathbb{R}_{+}^{n}\right) / \mathcal{A}_{[-1-\ell-n / p]}^{\Delta}$ as solution to (2.2).

For (2.3), the compatibility condition is

$$
\forall \psi \in \mathcal{A}_{\left[1+\ell-n / p^{\prime}\right]}^{\Delta}, \quad\left\langle z_{n}, \psi\right\rangle_{W_{\ell}^{-1, p}\left(\mathbb{R}_{+}^{n}\right) \times \stackrel{\circ}{W_{-\ell}^{1, p^{\prime}}\left(\mathbb{R}_{+}^{n}\right)}}=\int_{\Gamma} g_{n} \partial_{n} \psi \mathrm{d} x^{\prime}
$$

(see [5, Theorem 2.5]). First, (3.6) implies that for any $\psi \in \mathcal{A}_{\left[1+\ell-n / p^{\prime}\right]}^{\Delta}$, we have $\int_{\Gamma} g_{n} \partial_{n} \psi \mathrm{d} x^{\prime}=0$. It remains to show that the left-hand term is also zero. For this, we need to express $\mathcal{A}_{\left[1+\ell-n / p^{\prime}\right]}^{\Delta}$ by means of the kernel of the biharmonic operator $\mathcal{B}_{k}$ - that is the space of polynomials $\zeta$ such that $\Delta^{2} \zeta=0$ in $\mathbb{R}_{+}^{n}$ and $\zeta=\partial_{n} \zeta=0$ on $\Gamma$-. We showed in [5, Lemma 4.4] that

$$
\forall k \in \mathbb{Z}, \quad \mathcal{B}_{k+2}=\Pi_{D} \mathcal{A}_{k}^{\Delta} \oplus \Pi_{N} \mathcal{N}_{k}^{\Delta},
$$

where $\Pi_{D}$ - which is the equivalent for the odd harmonic polynomials with respect to $x_{n}$ of the operator $\Pi_{N}$ for the even harmonic polynomials with respect to $x_{n}$ - is defined as follows:

$$
\forall r \in \mathcal{A}_{k}^{\Delta}, \quad \Pi_{D} r\left(x^{\prime}, x_{n}\right)=\frac{1}{2} \int_{0}^{x_{n}} t r\left(x^{\prime}, t\right) \mathrm{d} t
$$

and satisfies for all $r \in \mathcal{A}_{k}^{\Delta}, \Delta \Pi_{D} r=r$ in $\mathbb{R}_{+}^{n}$ and $\Pi_{D} r=\partial_{n} \Pi_{D} r=0$ on $\Gamma$. From (3.9), we get for any $\psi \in \mathcal{A}_{\left[1+\ell-n / p^{\prime}\right]}^{\Delta}, \Pi_{D} \psi=\zeta \in \mathcal{B}_{\left[3+\ell-n / p^{\prime}\right]}$ and thus we have $\psi=\Delta \zeta$. So, by means of a Green formula (see [7, Lemma 3.7] for the justification), we get

$\forall \psi \in \mathcal{A}_{\left[1+\ell-n / p^{\prime}\right]}^{\Delta}, \exists \zeta \in \mathcal{B}_{\left[3+\ell-n / p^{\prime}\right]}$ such that

$$
\left\langle z_{n}, \psi\right\rangle_{W_{\ell}^{-1, p}\left(\mathbb{R}_{+}^{n}\right) \times \stackrel{\circ}{W_{-\ell}^{1, p^{\prime}}}\left(\mathbb{R}_{+}^{n}\right)}=\left\langle z_{n}, \Delta \zeta\right\rangle=\left\langle\Delta z_{n}, \zeta\right\rangle=0
$$

So (3.8) is proved and we get $u_{n} \in W_{\ell}^{1, p}\left(\mathbb{R}_{+}^{n}\right) / \mathcal{A}_{[1-\ell-n / p]}^{\Delta}$ as solution to (2.3).

Step 2: Problem (N1). Here $\Delta u_{n} \in W_{\ell}^{-1-1 / p, p}(\Gamma)$ (see [7, Lemma 3.7]) and for this problem, the compatibility condition is

$$
\forall \psi \in \mathcal{N}_{\left[2+\ell-n / p^{\prime}\right]}^{\Delta}, \quad\left\langle\Delta u_{n}, \psi\right\rangle_{W_{\ell}^{-1-1 / p, p}(\Gamma) \times W_{-\ell}^{2-1 / p^{\prime}, p^{\prime}}(\Gamma)}=0
$$

(see [7, Theorem 3.3]). For any $\psi \in \mathcal{N}_{\left[2+\ell-n / p^{\prime}\right]}^{\Delta}$, if we put $\zeta=\int_{0}^{x_{n}} \psi\left(x^{\prime}, t\right) \mathrm{d} t$, this yields $\psi=\partial_{n} \zeta$ with $\zeta \in \mathcal{A}_{\left[3+\ell-n / p^{\prime}\right]}^{\Delta}$. Since $\Delta u_{n}=\Delta^{\prime} g_{n}-\operatorname{div}^{\prime} \boldsymbol{g}^{\prime}$ on $\Gamma$, we see that (3.10) is exactly written as the condition (3.7), which is satisfied. 
So, we get $\pi \in W_{\ell}^{0, p}\left(\mathbb{R}_{+}^{n}\right) / \mathcal{N}_{[-\ell-n / p]}^{\Delta}$ as solution to $(N 1)$.

Step 3: Problem (N2). For (2.6), the compatibility condition is

$$
\forall \psi \in \mathcal{N}_{\left[\ell-n / p^{\prime}\right]}^{\Delta}, \quad \int_{\mathbb{R}_{+}^{n}} \pi \psi \mathrm{d} x=0
$$

(see [4, Theorem 3.1]). According to (3.9), we also have for any $\psi \in \mathcal{N}_{\left[\ell-n / p^{\prime}\right]}^{\Delta}$, $\Pi_{N} \psi=\zeta \in \mathcal{B}_{\left[2+\ell-n / p^{\prime}\right]}$ and thus $\psi=\Delta \zeta$. So, we have

$$
\begin{aligned}
& \forall \psi \in \mathcal{N}_{\left[\ell-n / p^{\prime}\right]}^{\Delta}, \exists \zeta \in \mathcal{B}_{\left[2+\ell-n / p^{\prime}\right]} \text { such that } \\
& \qquad \int_{\mathbb{R}_{+}^{n}} \pi \psi \mathrm{d} x=\int_{\mathbb{R}_{+}^{n}} \pi \Delta \zeta \mathrm{d} x=\langle\Delta \pi, \zeta\rangle=0 .
\end{aligned}
$$

Thus (3.11) is proved and we get $w \in W_{\ell}^{2, p}\left(\mathbb{R}_{+}^{n}\right) / \mathcal{N}_{[2-\ell-n / p]}^{\Delta}$ as solution to (2.6). Consequently, $\boldsymbol{v}^{\prime}=\nabla^{\prime} w \in \boldsymbol{W}_{\ell}^{1, p}\left(\mathbb{R}_{+}^{n}\right) / \mathcal{N}_{[1-\ell-n / p]}^{\Delta}$ is solution to problem (2.4).

Finally, for problem (2.5), the compatibility condition is

$$
\forall \boldsymbol{\varphi}^{\prime} \in \mathcal{N}_{\left[1+\ell-n / p^{\prime}\right]}^{\Delta}, \quad\left\langle\boldsymbol{g}^{\prime}, \boldsymbol{\varphi}^{\prime}\right\rangle_{\boldsymbol{W}_{\ell}^{-1 / p, p}(\Gamma) \times \boldsymbol{W}_{-\ell}^{1-1 / p^{\prime}, p^{\prime}}(\Gamma)}=0
$$

(see [7, Theorem 3.4]). It is clear that (3.12) is include in (3.6) and then we get $\boldsymbol{z}^{\prime} \in \boldsymbol{W}_{\ell}^{1, p}\left(\mathbb{R}_{+}^{n}\right) / \mathcal{N}_{[1-\ell-n / p]}^{\Delta}$ as solution to $(2.5)$.

So $\boldsymbol{u}^{\prime}=\boldsymbol{v}^{\prime}+\boldsymbol{z}^{\prime} \in \boldsymbol{W}_{\ell}^{1, p}\left(\mathbb{R}_{+}^{n}\right) / \mathcal{N}_{[1-\ell-n / p]}^{\Delta}$ is solution to $(N 2)$.

Remark 3.6. The set of critical values for all these auxiliary problems is given by hypothesis (1.6). This is the good set of critical values for the homogeneous problem (2.1) and the supplementary value $n / p^{\prime}=\ell+1$ will only appear in the nonhomogeneous problem $\left(S^{\sharp}\right)$.

(iii) To recover the $n^{\text {th }}$ component of $(2.1 \mathrm{a})$ - that is $(2.7)$ - and (2.1b) from $(B),(N 1)$ and $(N 2)$, we will use the nonuniqueness of their respective solutions $u_{n}, \pi$ and $\boldsymbol{u}^{\prime}$ constructed in (ii), to select a "good one".

Since $\Delta u_{n}-\partial_{n} \pi$ satisfies $(2.8)$ and belongs to $W_{\ell}^{-1, p}\left(\mathbb{R}_{+}^{n}\right)$, we can deduce that $\Delta u_{n}-\partial_{n} \pi \in \mathcal{A}_{[-1-\ell-n / p]}^{\Delta}$. As $\pi$ is defined up to an element of $\mathcal{N}_{[-\ell-n / p]}^{\Delta}, \partial_{n} \pi$ is defined up to an element of $\mathcal{A}_{[-1-\ell-n / p]}^{\Delta}$ and thus we can chose $\pi$ such that $\Delta u_{n}-\partial_{n} \pi=0$.

Since $\operatorname{div} \boldsymbol{u}$ satisfies (2.9) and belongs to $W_{\ell}^{0, p}\left(\mathbb{R}_{+}^{n}\right)$, we can deduce that $\operatorname{div} \boldsymbol{u} \in$ $\mathcal{N}_{[-\ell-n / p]}^{\Delta}$. As $\boldsymbol{u}^{\prime}$ is defined up to an element of $\mathcal{N}_{[1-\ell-n / p]}^{\Delta}, \operatorname{div}^{\prime} \boldsymbol{u}^{\prime}$ is defined up to an element of $\mathcal{N}_{[-\ell-n / p]}^{\Delta}$ and thus we can chose $\boldsymbol{u}^{\prime}$ such that div $\boldsymbol{u}=0$.

To finish this proof, let us notice that the characterization of the kernel answers to point (iv).

So we have established the existence of weak solutions to the homogeneous problem and we can sum up that in the following result:

Proposition 3.7. Let $\ell \in \mathbb{Z}$ with hypothesis (1.6). For any $g_{n} \in W_{\ell}^{1-1 / p, p}(\Gamma)$ and $\boldsymbol{g}^{\prime} \in \boldsymbol{W}_{\ell}^{-1 / p, p}(\Gamma)$, satisfying the compatibility condition (3.6), problem (2.1) admits a solution $(\boldsymbol{u}, \pi) \in \boldsymbol{W}_{\ell}^{1, p}\left(\mathbb{R}_{+}^{n}\right) \times W_{\ell}^{0, p}\left(\mathbb{R}_{+}^{n}\right)$, unique up to an element of $\mathcal{S}_{[1-\ell-n / p]}^{\sharp}$, with the estimate

$$
\begin{gathered}
\inf _{(\boldsymbol{\lambda}, \mu) \in \mathcal{S}_{[1-\ell-n / p]}^{\sharp}}\left(\|\boldsymbol{u}+\boldsymbol{\lambda}\|_{\boldsymbol{W}_{\ell}^{1, p}\left(\mathbb{R}_{+}^{n}\right)}+\|\pi+\mu\|_{W_{\ell}^{0, p}\left(\mathbb{R}_{+}^{n}\right)}\right) \leqslant \\
C\left(\left\|g_{n}\right\|_{W_{\ell}^{1-1 / p, p}(\Gamma)}+\left\|\boldsymbol{g}^{\prime}\right\|_{\boldsymbol{W}_{\ell}^{-1 / p, p}(\Gamma)}\right) .
\end{gathered}
$$


Now, always for the homogeneous problem, we will consider the case of more regular boundary conditions, which yields strong solutions.

Proposition 3.8. Let $\ell \in \mathbb{Z}$ and assume that

$$
n / p^{\prime} \notin\{1, \ldots, \ell+1\} \quad \text { and } \quad n / p \notin\{1, \ldots,-\ell-1\} .
$$

For any $g_{n} \in W_{\ell+1}^{2-1 / p, p}(\Gamma)$ and $\boldsymbol{g}^{\prime} \in \boldsymbol{W}_{\ell+1}^{1-1 / p, p}(\Gamma)$, satisfying the condition (3.6), problem (2.1) admits a solution $(\boldsymbol{u}, \pi) \in \boldsymbol{W}_{\ell+1}^{2, p}\left(\mathbb{R}_{+}^{n}\right) \times W_{\ell+1}^{1, p}\left(\mathbb{R}_{+}^{n}\right)$, unique up to an element of $\mathcal{S}_{[1-\ell-n / p]}^{\sharp}$, with the corresponding estimate.

Proof. We simply resume the proof of Proposition 3.7 - which we named "Proof of Proposition 2.1 revisited" - at the begining of Subsection 3.4, using the regularity results for the harmonic and biharmonic operators.

In order, for the biharmonic problem $(B)$, split in the Dirichlet problems $(2.2)$ and (2.3), we find $z_{n} \in W_{\ell+1}^{0, p}\left(\mathbb{R}_{+}^{n}\right)$ solution to (2.2) (see [7, Theorem 3.8]); as well we find $u_{n} \in W_{\ell+1}^{2, p}\left(\mathbb{R}_{+}^{n}\right)$ solution to (2.3) (see [3, Corollary 3.4]). For the first Neumann problem $(N 1)$, we find $\pi \in W_{\ell+1}^{1, p}\left(\mathbb{R}_{+}^{n}\right)$ (see [7, Theorem 3.4]). Last, concerning the second Neumann problem (N2), we find $\boldsymbol{u}^{\prime} \in \boldsymbol{W}_{\ell+1}^{2, p}\left(\mathbb{R}_{+}^{n}\right)$ (see [4, Corollary 3.3]). Moreover, all these results hold under hypothesis (3.13), which yields the optimal set of critical values for such data.

Remark 3.9. We also can get Proposition 3.8 as a regularity result from Proposition 3.7. Indeed, we have $W_{\ell+1}^{2-1 / p, p}(\Gamma) \hookrightarrow W_{\ell}^{1-1 / p, p}(\Gamma)$ if $\frac{n}{p} \neq-\ell$. On the other hand, the imbedding $W_{\ell+1}^{1-1 / p, p}(\Gamma) \hookrightarrow W_{\ell}^{-1 / p, p}(\Gamma)$ can be broken down into $W_{\ell+1}^{1-1 / p, p}(\Gamma) \hookrightarrow W_{\ell+1 / p}^{0, p}(\Gamma)$ and $W_{\ell+1 / p}^{0, p}(\Gamma) \hookrightarrow W_{\ell}^{-1 / p, p}(\Gamma)$. The first one also holds if $\frac{n}{p} \neq-\ell$ and by duality, we find $\frac{n}{p^{\prime}} \neq \ell+1$ as condition for the second one. So, under hypothesis (3.13), if in addition $\frac{n}{p} \neq-\ell$, we can deduce from Proposition 3.7 that problem $(2.1)$ admits a solution $(\boldsymbol{u}, \pi) \in \boldsymbol{W}_{\ell}^{1, p}\left(\mathbb{R}_{+}^{n}\right) \times W_{\ell}^{0, p}\left(\mathbb{R}_{+}^{n}\right)$. Then, as in [7, Corollary 5.5], we can show by regularity arguments that in fact $(\boldsymbol{u}, \pi) \in \boldsymbol{W}_{\ell+1}^{2, p}\left(\mathbb{R}_{+}^{n}\right) \times W_{\ell+1}^{1, p}\left(\mathbb{R}_{+}^{n}\right)$. The cost of this approach is thus the supplementary critical value $\frac{n}{p}=-\ell$.

3.5. The nonhomogeneous case. We start with enough regular data on the boundary - that is the ones of Proposition 3.8 - to get strong solutions to the complete problem.

THeOREM 3.10. Let $\ell \in \mathbb{Z}$ with hypothesis (3.13). For any $\boldsymbol{f} \in \boldsymbol{W}_{\ell+1}^{0, p}\left(\mathbb{R}_{+}^{n}\right)$, $h \in W_{\ell+1}^{1, p}\left(\mathbb{R}_{+}^{n}\right), g_{n} \in W_{\ell+1}^{2-1 / p, p}(\Gamma), \boldsymbol{g}^{\prime} \in \boldsymbol{W}_{\ell+1}^{1-1 / p, p}(\Gamma)$, satisfying the condition (3.4), problem $\left(S^{\sharp}\right)$ admits a solution $(\boldsymbol{u}, \pi) \in \boldsymbol{W}_{\ell+1}^{2, p}\left(\mathbb{R}_{+}^{n}\right) \times W_{\ell+1}^{1, p}\left(\mathbb{R}_{+}^{n}\right)$, unique up to an element of $\mathcal{S}_{[1-\ell-n / p]}^{\sharp}$, with the estimate

$$
\begin{aligned}
& \inf _{(\boldsymbol{\lambda}, \mu) \in \mathcal{S}_{[1-\ell-n / p]}^{\sharp}}\left(\|\boldsymbol{u}+\boldsymbol{\lambda}\|_{\boldsymbol{W}_{\ell+1}^{2, p}\left(\mathbb{R}_{+}^{n}\right)}+\|\pi+\mu\|_{W_{\ell+1}^{1, p}\left(\mathbb{R}_{+}^{n}\right)}\right) \leqslant \\
& C\left(\|\boldsymbol{f}\|_{\boldsymbol{W}_{\ell+1}^{0, p}\left(\mathbb{R}_{+}^{n}\right)}+\|h\|_{W_{\ell+1}^{0, p}\left(\mathbb{R}_{+}^{n}\right)}+\left\|g_{n}\right\|_{W_{\ell+1}^{2-1 / p, p}(\Gamma)}+\left\|\boldsymbol{g}^{\prime}\right\|_{\boldsymbol{W}_{\ell+1}^{1-1 / p, p}(\Gamma)}\right) .
\end{aligned}
$$

Proof. It suffices to show the existence. We will naturally use the result in the homogeneous case established above and first, we consider the lifted problem

$$
\left(S_{b}\right)\left\{\begin{array}{rlll}
-\Delta \boldsymbol{v}+\nabla \pi=\boldsymbol{F} & \text { and } & \operatorname{div} \boldsymbol{v}=H & \text { in } \mathbb{R}_{+}^{n}, \\
v_{n}=0 & \text { and } \quad \partial_{n} \boldsymbol{v}^{\prime}=\mathbf{0} & \text { on } \Gamma .
\end{array}\right.
$$


Indeed, according to Lemma 1.1, there exists a lifting function $\boldsymbol{u}_{\boldsymbol{g}^{\prime}} \in \boldsymbol{W}_{\ell+1}^{2, p}\left(\mathbb{R}_{+}^{n}\right)$ of $\boldsymbol{g}^{\prime}$ such that $\partial_{n} \boldsymbol{u}_{\boldsymbol{g}^{\prime}}=\boldsymbol{g}^{\prime}$ on $\Gamma$ and there also exists $u_{g_{n}} \in W_{\ell+1}^{2, p}\left(\mathbb{R}_{+}^{n}\right)$ such that $u_{g_{n}}=g_{n}$ on $\Gamma$. Then, if we put $\boldsymbol{u}_{\boldsymbol{g}}=\left(\boldsymbol{u}_{\boldsymbol{g}^{\prime}}, u_{g_{n}}\right), \boldsymbol{F}=\boldsymbol{f}+\Delta \boldsymbol{u}_{\boldsymbol{g}}, H=h-\operatorname{div} \boldsymbol{u}_{\boldsymbol{g}}$ and $\boldsymbol{v}=\boldsymbol{u}-\boldsymbol{u}_{\boldsymbol{g}}$, the two problems $\left(S_{\mathrm{b}}\right)$ and $\left(S^{\sharp}\right)$ are equivalent. In addition, by means of Green formulae, we can easily verify that condition $(3.5)$ - i.e. the alternative form of $(3.4)$ - becomes for $\left(S_{\mathrm{b}}\right)$ :

$$
\forall(\boldsymbol{\lambda}, \mu) \in \mathcal{S}_{\left[1+\ell-n / p^{\prime}\right]}^{\sharp}, \quad \int_{\mathbb{R}_{+}^{n}} \boldsymbol{F} \cdot \boldsymbol{\lambda} \mathrm{d} x-\int_{\mathbb{R}_{+}^{n}} H \mu \mathrm{d} x=0 .
$$

Next, we extend $\boldsymbol{F}$ and $H$ to the whole space by $\tilde{\boldsymbol{F}} \in \boldsymbol{W}_{\ell+1}^{0, p}\left(\mathbb{R}^{n}\right)$ and $\tilde{H} \in W_{\ell+1}^{1, p}\left(\mathbb{R}^{n}\right)$ as follows:

$$
\begin{aligned}
& \forall \boldsymbol{\varphi} \in \mathcal{D}\left(\mathbb{R}^{n}\right), \forall \psi \in \mathcal{D}\left(\mathbb{R}^{n}\right), \\
& \int_{\mathbb{R}^{n}} \tilde{\boldsymbol{F}} \cdot \boldsymbol{\varphi} \mathrm{d} x=\int_{\mathbb{R}_{+}^{n}} \boldsymbol{F} \cdot\left(\boldsymbol{\varphi}^{\prime}+\boldsymbol{\varphi}^{\prime *}, \varphi_{n}-\varphi_{n}^{*}\right) \mathrm{d} x, \\
& \int_{\mathbb{R}^{n}} \tilde{H} \psi \mathrm{d} x=\int_{\mathbb{R}_{+}^{n}} H\left(\psi+\psi^{*}\right) \mathrm{d} x,
\end{aligned}
$$

where $\psi^{*}(x)=\psi\left(x^{*}\right)$ for any $x=\left(x^{\prime}, x_{n}\right) \in \mathbb{R}^{n}$ with $x^{*}=\left(x^{\prime},-x_{n}\right)$. That is, to give the functional writing to this extension:

$$
(\tilde{\boldsymbol{F}}, \tilde{H})\left(x^{\prime}, x_{n}\right)= \begin{cases}(\boldsymbol{F}, H)\left(x^{\prime}, x_{n}\right) & \text { if } x_{n}>0 \\ \left(\boldsymbol{F}^{\prime},-F_{n}, H\right)\left(x^{\prime},-x_{n}\right) & \text { if } x_{n}<0 .\end{cases}
$$

Now, by Theorem 1.3, with hypothesis (3.13), we know that there exists $(\boldsymbol{w}, \vartheta) \in$ $\boldsymbol{W}_{\ell+1}^{2, p}\left(\mathbb{R}^{n}\right) \times W_{\ell+1}^{1, p}\left(\mathbb{R}^{n}\right)$ solution to the problem

$$
(\tilde{S}): \quad-\Delta \boldsymbol{w}+\nabla \vartheta=\tilde{\boldsymbol{F}} \text { and } \operatorname{div} \boldsymbol{w}=\tilde{H} \text { in } \mathbb{R}^{n},
$$

provided the condition $(\tilde{\boldsymbol{F}}, \tilde{H}) \perp \mathcal{S}_{\left[1+\ell-n / p^{\prime}\right]}$ is fulfilled, that is

$$
\forall(\boldsymbol{\Lambda}, M) \in \mathcal{S}_{\left[1+\ell-n / p^{\prime}\right]}, \quad \int_{\mathbb{R}^{n}} \tilde{\boldsymbol{F}} \cdot \boldsymbol{\Lambda} \mathrm{d} x-\int_{\mathbb{R}^{n}} \tilde{H} M \mathrm{~d} x=0 .
$$

Thanks to (3.15), we can write (3.16) as

$$
\int_{\mathbb{R}_{+}^{n}} \boldsymbol{F} \cdot\left(\boldsymbol{\Lambda}^{\prime}+\boldsymbol{\Lambda}^{\prime *}, \Lambda_{n}-\Lambda_{n}^{*}\right) \mathrm{d} x-\int_{\mathbb{R}_{+}^{n}} H\left(M+M^{*}\right) \mathrm{d} x=0 .
$$

Since $\left(\boldsymbol{\Lambda}^{\prime}+\boldsymbol{\Lambda}^{\prime *}, \Lambda_{n}-\Lambda_{n}^{*}, M+M^{*}\right) \in \mathcal{S}_{\left[1+\ell-n / p^{\prime}\right]}^{\sharp}$, the condition (3.17) - and thus (3.16) - is a simple consequence of (3.14). Then, the pair of functions $(\boldsymbol{v}, \pi)$ defined in $\mathbb{R}_{+}^{n}$ by

$$
(\boldsymbol{v}, \pi)=\frac{1}{2}\left(\boldsymbol{w}^{\prime}+\boldsymbol{w}^{\prime *}, w_{n}-w_{n}^{*}, \vartheta+\vartheta^{*}\right)
$$

belongs to $\boldsymbol{W}_{\ell+1}^{2, p}\left(\mathbb{R}_{+}^{n}\right) \times W_{\ell+1}^{1, p}\left(\mathbb{R}_{+}^{n}\right)$ and, by a straightforward calculation, we can see that it satisfies $\left(S_{b}\right)$.

Now, we can establish the existence of generalized solutions announced in Subsection 3.2 . 
Proof of the existence in Theorem 3.5. (i) Assume that $\frac{n}{p^{\prime}}>\ell+1$. According to Proposition 3.7 , there exists $(\boldsymbol{v}, \vartheta) \in \boldsymbol{W}_{\ell}^{1, p}\left(\mathbb{R}_{+}^{n}\right) \times W_{\ell}^{0, p}\left(\mathbb{R}_{+}^{n}\right)$ satisfying

$$
\left\{\begin{array}{rlll}
-\Delta \boldsymbol{v}+\nabla \vartheta=\mathbf{0} & \text { and } & \operatorname{div} \boldsymbol{v}=0 & \text { in } \mathbb{R}_{+}^{n} \\
v_{n}=g_{n} & \text { and } & \partial_{n} \boldsymbol{v}^{\prime}=\boldsymbol{g}^{\prime} & \text { on } \Gamma .
\end{array}\right.
$$

In addition, by Theorem 3.10 , there exists $(\boldsymbol{w}, \zeta) \in \boldsymbol{W}_{\ell+1}^{2, p}\left(\mathbb{R}_{+}^{n}\right) \times W_{\ell+1}^{1, p}\left(\mathbb{R}_{+}^{n}\right)$ satisfying

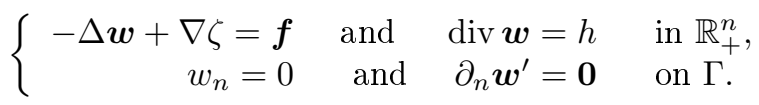

The pair $(\boldsymbol{u}, \pi)=(\boldsymbol{v}+\boldsymbol{w}, \vartheta+\zeta)$ answers to the question.

(ii) Assume that $\frac{n}{p^{\prime}}<\ell+1$. We cannot directly construct a solution as above. Indeed, the compatibility conditions — which are now non-trivial — of the auxiliary and initial problems must coincide. Let $N$ be the dimension of the subspace $\mathcal{S}_{\left[1+\ell-n / p^{\prime}\right]}^{\sharp}$ of $\boldsymbol{W}_{-\ell+1}^{2, p^{\prime}}\left(\mathbb{R}_{+}^{n}\right) \times W_{-\ell+1}^{1, p^{\prime}}\left(\mathbb{R}_{+}^{n}\right)$, which is imbedded in $\boldsymbol{W}_{-\ell-1}^{0, p^{\prime}}\left(\mathbb{R}_{+}^{n}\right) \times W_{-\ell-1}^{-1, p^{\prime}}\left(\mathbb{R}_{+}^{n}\right)$ and $\left\{\boldsymbol{e}_{1}, \ldots, \boldsymbol{e}_{N}\right\}$ a basis of $\mathcal{S}_{\left[1+\ell-n / p^{\prime}\right]}^{\sharp}$. According to Hahn-Banach Theorem, there exists a family $\left\{\boldsymbol{e}_{1}^{*}, \ldots, \boldsymbol{e}_{N}^{*}\right\}$ of elements of $\boldsymbol{W}_{\ell+1}^{0, p}\left(\mathbb{R}_{+}^{n}\right) \times W_{\ell+1}^{1, p}\left(\mathbb{R}_{+}^{n}\right)$, which extends the dual basis of the dual space $\left(\mathcal{S}_{\left[1+\ell-n / p^{\prime}\right]}^{\sharp}\right)^{\prime}$. First, we can give a more compact writing of the compatibility condition $(3.5)$ - which is equivalent to $(3.4)$ - as:

$$
\begin{aligned}
\forall(\boldsymbol{\lambda}, \mu) \in & \mathcal{S}_{\left[1+\ell-n / p^{\prime}\right]}^{\sharp}, \\
& \langle(\boldsymbol{f},-h),(\boldsymbol{\lambda}, \mu)\rangle_{\boldsymbol{W}_{\ell+1}^{0, p}\left(\mathbb{R}_{+}^{n}\right) \times W_{\ell}^{0, p}\left(\mathbb{R}_{+}^{n}\right), \boldsymbol{W}_{-\ell-1}^{0, p^{\prime}}\left(\mathbb{R}_{+}^{n}\right) \times W_{-\ell}^{0, p^{\prime}}\left(\mathbb{R}_{+}^{n}\right)}= \\
& \left\langle\boldsymbol{g},\left(\boldsymbol{\lambda}^{\prime}, \mu-\partial_{n} \lambda_{n}\right)\right\rangle_{\boldsymbol{W}_{\ell}^{-1 / p, p}(\Gamma) \times W_{\ell}^{1-1 / p, p}(\Gamma), \boldsymbol{W}_{-\ell}^{1-1 / p^{\prime}, p^{\prime}}(\Gamma) \times W_{-\ell}^{-1 / p^{\prime}, p^{\prime}}(\Gamma)},
\end{aligned}
$$

where $\boldsymbol{g}=\left(\boldsymbol{g}^{\prime}, g_{n}\right)$. We denote the corresponding trace mapping by

$$
\begin{aligned}
\kappa: \boldsymbol{W}_{-\ell+1}^{2, p^{\prime}}\left(\mathbb{R}_{+}^{n}\right) \times W_{-\ell+1}^{1, p^{\prime}}\left(\mathbb{R}_{+}^{n}\right) & \longrightarrow \boldsymbol{W}_{-\ell+1}^{2-1 / p^{\prime}, p^{\prime}}(\Gamma) \times W_{-\ell+1}^{1-1 / p^{\prime}, p^{\prime}}(\Gamma) \\
(\boldsymbol{\lambda}, \mu) & \longmapsto\left(\gamma_{0} \boldsymbol{\lambda}^{\prime}, \gamma_{0} \mu-\gamma_{1} \lambda_{n}\right)
\end{aligned}
$$

and $\varepsilon_{i}=\kappa\left(\boldsymbol{e}_{i}\right)$. With a suitable numbering of the family, $\left\{\varepsilon_{1}, \ldots, \varepsilon_{d}\right\}$ form a basis of the subspace $\kappa\left(\mathcal{S}_{\left[1+\ell-n / p^{\prime}\right]}^{\sharp}\right)$ of $\boldsymbol{W}_{-\ell+1}^{2-1 / p^{\prime}, p^{\prime}}(\Gamma) \times W_{-\ell+1}^{1-1 / p^{\prime}, p^{\prime}}(\Gamma) \hookrightarrow \boldsymbol{W}_{-\ell-1}^{-1 / p^{\prime}, p^{\prime}}(\Gamma) \times$ $W_{-\ell-1}^{-1-1 / p^{\prime}, p^{\prime}}(\Gamma)$ and $\varepsilon_{i}=\mathbf{0}$ for $i \in\{d+1, \ldots, N\}$. Here again, according to HahnBanach Theorem, there exists a family $\left\{\varepsilon_{1}^{*}, \ldots, \varepsilon_{d}^{*}\right\}$ of elements of $\boldsymbol{W}_{\ell+1}^{1-1 / p, p}(\Gamma) \times$ $W_{\ell+1}^{2-1 / p, p}(\Gamma)$ which extends the dual basis of $\left\{\varepsilon_{1}, \ldots, \varepsilon_{d}\right\}$. Now, let us consider the functions defined by

$$
(\boldsymbol{F},-H)=\sum_{i=1}^{N} \boldsymbol{e}_{i}^{*}\left\langle(\boldsymbol{f},-h), \boldsymbol{e}_{i}\right\rangle \text { and } \boldsymbol{G}=\sum_{i=1}^{d} \varepsilon_{i}^{*}\left\langle\boldsymbol{g}, \boldsymbol{\varepsilon}_{i}\right\rangle .
$$

They satisfy

$$
\begin{aligned}
& \left\langle(\boldsymbol{F},-H), e_{k}\right\rangle=\left\langle(\boldsymbol{f},-h), e_{k}\right\rangle=\left\langle\boldsymbol{g}, \boldsymbol{\varepsilon}_{k}\right\rangle \text { for } k \in\{1, \ldots, N\} \\
& \left\langle(\boldsymbol{F},-H), e_{k}\right\rangle=\left\langle\boldsymbol{G}, \boldsymbol{\varepsilon}_{k}\right\rangle=\left\langle\boldsymbol{g}, \boldsymbol{\varepsilon}_{k}\right\rangle \text { for } k \in\{1, \ldots, d\} \\
& \left\langle(\boldsymbol{F},-H), e_{k}\right\rangle=\left\langle\boldsymbol{G}, \boldsymbol{\varepsilon}_{k}\right\rangle=0 \text { for } k \in\{d+1, \ldots, N\}
\end{aligned}
$$


By Theorem 3.10, there exists $(\boldsymbol{v}, \vartheta) \in \boldsymbol{W}_{\ell+1}^{2, p}\left(\mathbb{R}_{+}^{n}\right) \times W_{\ell+1}^{1, p}\left(\mathbb{R}_{+}^{n}\right)$ satisfying

$$
\left\{\begin{aligned}
(-\Delta \boldsymbol{v}+\nabla \vartheta, \operatorname{div} \boldsymbol{v}) & =(\boldsymbol{f}-\boldsymbol{F}, h-H) & & \text { in } \mathbb{R}_{+}^{n}, \\
\left(\partial_{n} \boldsymbol{v}^{\prime}, v_{n}\right) & =\mathbf{0} & & \text { on } \Gamma .
\end{aligned}\right.
$$

By Proposition 3.7, there exists $(\boldsymbol{w}, \zeta) \in \boldsymbol{W}_{\ell}^{1, p}\left(\mathbb{R}_{+}^{n}\right) \times W_{\ell}^{0, p}\left(\mathbb{R}_{+}^{n}\right)$ satisfying

$$
\left\{\begin{aligned}
(-\Delta \boldsymbol{w}+\nabla \zeta, \operatorname{div} \boldsymbol{w}) & =\mathbf{0} & & \text { in } \mathbb{R}_{+}^{n}, \\
\left(\partial_{n} \boldsymbol{w}^{\prime}, w_{n}\right) & =\boldsymbol{g}-\boldsymbol{G} & & \text { on } \Gamma .
\end{aligned}\right.
$$

By Theorem 3.10, there exists $(\boldsymbol{z}, \eta) \in \boldsymbol{W}_{\ell+1}^{2, p}\left(\mathbb{R}_{+}^{n}\right) \times W_{\ell+1}^{1, p}\left(\mathbb{R}_{+}^{n}\right)$ satisfying

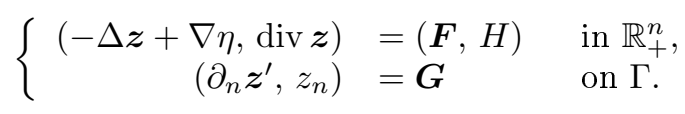

Finally, the pair $(\boldsymbol{u}, \pi)=(\boldsymbol{v}+\boldsymbol{w}+\boldsymbol{z}, \vartheta+\zeta+\eta)$ answers to the question.

To end this section, we can give a global regularity result which extends the strong solutions of Theorem 3.10.

Corollary 3.11. Let $\ell \in \mathbb{Z}$ and $m \geqslant 1$ be two integers and assume (1.7). For all $\boldsymbol{f} \in \boldsymbol{W}_{m+\ell}^{m-1, p}\left(\mathbb{R}_{+}^{n}\right), h \in W_{m+\ell}^{m, p}\left(\mathbb{R}_{+}^{n}\right), g_{n} \in W_{m+\ell}^{m+1-1 / p, p}(\Gamma)$ and $\boldsymbol{g}^{\prime} \in \boldsymbol{W}_{m+\ell}^{m-1 / p, p}(\Gamma)$, satisfying the compatibility condition $(3.4)$, problem $\left(S^{\sharp}\right)$ admits a solution $(\boldsymbol{u}, \pi) \in$ $\boldsymbol{W}_{m+\ell}^{m+1, p}\left(\mathbb{R}_{+}^{n}\right) \times W_{m+\ell}^{m, p}\left(\mathbb{R}_{+}^{n}\right)$, unique up to an element of $\mathcal{S}_{[1-\ell-n / p]}^{\sharp}$, with the estimate

$$
\begin{aligned}
& \inf _{(\boldsymbol{\lambda}, \mu) \in \mathcal{S}_{[1-\ell-n / p]}^{\sharp}}\left(\|\boldsymbol{u}+\boldsymbol{\lambda}\|_{\boldsymbol{W}_{m+\ell}^{m+1, p}\left(\mathbb{R}_{+}^{n}\right)}+\|\pi+\mu\|_{W_{m+\ell}^{m, p}\left(\mathbb{R}_{+}^{n}\right)}\right) \leqslant \\
& C\left(\|\boldsymbol{f}\|_{\boldsymbol{W}_{m+\ell}^{m-1, p}\left(\mathbb{R}_{+}^{n}\right)}+\|h\|_{W_{m+\ell}^{m, p}\left(\mathbb{R}_{+}^{n}\right)}+\left\|g_{n}\right\|_{W_{m+\ell}^{m+1-1 / p, p}(\Gamma)}+\left\|\boldsymbol{g}^{\prime}\right\|_{\boldsymbol{W}_{m+\ell}^{m-1 / p, p}(\Gamma)}\right) .
\end{aligned}
$$

Proof. The case $m=1$ corresponds to Theorem 3.10. We suppose that $m \geqslant 2$.

(1) Assuming that $\ell \leqslant-2$, hypothesis (1.7) — which yields the set of critical values - is reduced to $n / p \notin\{1, \ldots,-\ell-m\}$. We begin to establish the result for the homogeneous problem, as in Proposition 3.8. The arguments are the same, using the regularity results for the Laplacian with Dirichlet and Neumann boundary conditions (see $[3,5]$ ). Next, for the complete problem, we apply the method of the proof of Theorem 3.10 with an ad hoc extention for $\boldsymbol{F}$ and $H$ - in this case, there is no compatibility condition -

(2) Assuming that $\ell \geqslant-1$ and $n / p^{\prime} \notin\{1, \ldots, \ell+1\}$, we can adapt the proof by induction of the regularity result for the Stokes system with Dirichlet boundary conditions (see [7, Corollary 5.5]).

4. Very weak solutions. The aim of this section is to return to the homogeneous problem (2.1) in which we envisage now very singular data on the boundary, that is more precisely

$$
\boldsymbol{g}^{\prime} \in \boldsymbol{W}_{\ell-1}^{-1-1 / p, p}(\Gamma) \text { and } g_{n} \in W_{\ell-1}^{-1 / p, p}(\Gamma) .
$$

First, we will establish two preliminary lemmas. The second one yields a Green formula in order to solve this new problem by a duality argument.

Let us denote by

$$
T:(\boldsymbol{u}, \pi) \longmapsto(-\Delta \boldsymbol{u}+\nabla \pi,-\operatorname{div} \boldsymbol{u})
$$


the Stokes operator. For any $\ell \in \mathbb{Z}$, we introduce the space

$$
\begin{aligned}
& T_{\ell, 1}^{p}\left(\mathbb{R}_{+}^{n}\right)=\left\{(\boldsymbol{u}, \pi) \in \boldsymbol{W}_{\ell-1}^{0, p}\left(\mathbb{R}_{+}^{n}\right) \times W_{\ell-1}^{-1, p}\left(\mathbb{R}_{+}^{n}\right)\right. \\
&\left.T(\boldsymbol{u}, \pi) \in \boldsymbol{W}_{\ell+1,1}^{0, p}\left(\mathbb{R}_{+}^{n}\right) \times W_{\ell, 1}^{0, p}\left(\mathbb{R}_{+}^{n}\right)\right\},
\end{aligned}
$$

which is a reflexive Banach space equipped with the graph-norm. Then we have the following density result:

Lemma 4.1. Let $\ell \in \mathbb{Z}$ and assume that

$$
n / p^{\prime} \notin\{1, \ldots, \ell-1\} \quad \text { and } \quad n / p \notin\{1, \ldots,-\ell+1\}
$$

then the space $\mathcal{D}\left(\overline{\mathbb{R}_{+}^{n}}\right) \times \mathcal{D}\left(\overline{\mathbb{R}_{+}^{n}}\right)$ is dense in $T_{\ell, 1}^{p}\left(\mathbb{R}_{+}^{n}\right)$.

Proof. For every continuous linear form $\Lambda \in\left(T_{\ell, 1}^{p}\left(\mathbb{R}_{+}^{n}\right)\right)^{\prime}$, there exists a unique $(\boldsymbol{v}, \zeta, \boldsymbol{w}, \vartheta) \in \boldsymbol{W}_{-\ell+1}^{0, p^{\prime}}\left(\mathbb{R}_{+}^{n}\right) \times \stackrel{\circ}{W_{-\ell+1}^{1, p^{\prime}}}\left(\mathbb{R}_{+}^{n}\right) \times \boldsymbol{W}_{-\ell-1,-1}^{0, p^{\prime}}\left(\mathbb{R}_{+}^{n}\right) \times W_{-\ell,-1}^{0, p^{\prime}}\left(\mathbb{R}_{+}^{n}\right)$ such that for all $(\boldsymbol{u}, \pi) \in T_{\ell, 1}^{p}\left(\mathbb{R}_{+}^{n}\right)$,

$$
\langle\Lambda,(\boldsymbol{u}, \pi)\rangle=\langle(\boldsymbol{v}, \zeta),(\boldsymbol{u}, \pi)\rangle+\langle(\boldsymbol{w}, \vartheta), T(\boldsymbol{u}, \pi)\rangle .
$$

Thanks to the Hahn-Banach theorem, it suffices to show that any $\Lambda$ which vanishes on $\mathcal{D}\left(\overline{\mathbb{R}_{+}^{n}}\right) \times \mathcal{D}\left(\overline{\mathbb{R}_{+}^{n}}\right)$ is actually zero on $T_{\ell, 1}^{p}\left(\mathbb{R}_{+}^{n}\right)$. Let us suppose that $\Lambda=0$ on $\mathcal{D}\left(\overline{\mathbb{R}_{+}^{n}}\right) \times \mathcal{D}\left(\overline{\mathbb{R}_{+}^{n}}\right)$, thus on $\mathcal{D}\left(\mathbb{R}_{+}^{n}\right) \times \mathcal{D}\left(\mathbb{R}_{+}^{n}\right)$. Then we can deduce from (4.2) that

$$
(\boldsymbol{v}, \zeta)+T(\boldsymbol{w}, \vartheta)=0 \quad \text { in } \mathbb{R}_{+}^{n},
$$

hence $T(\boldsymbol{w}, \vartheta) \in \boldsymbol{W}_{-\ell+1}^{0, p^{\prime}}\left(\mathbb{R}_{+}^{n}\right) \times \stackrel{\circ}{W_{-\ell+1}^{1, p^{\prime}}}\left(\mathbb{R}_{+}^{n}\right)$. Let $\tilde{\boldsymbol{v}}, \tilde{\zeta}, \tilde{\boldsymbol{w}}, \tilde{\vartheta}$ be respectively the zero extensions of $\boldsymbol{v}, \zeta, \boldsymbol{w}, \vartheta$ to $\mathbb{R}^{n}$. By (4.2), it is clear that we have

$$
(\tilde{\boldsymbol{v}}, \tilde{\zeta})+T(\tilde{\boldsymbol{w}}, \tilde{\vartheta})=0 \text { in } \mathbb{R}^{n}
$$

and thus $T(\tilde{\boldsymbol{w}}, \tilde{\vartheta}) \in \boldsymbol{W}_{-\ell+1}^{0, p^{\prime}}\left(\mathbb{R}^{n}\right) \times W_{-\ell+1}^{1, p^{\prime}}\left(\mathbb{R}^{n}\right)$. Besides, we have the following Green formula: for any $(\varphi, \psi) \in \mathcal{D}\left(\mathbb{R}^{n}\right) \times \mathcal{D}\left(\mathbb{R}^{n}\right)$,

$$
\langle T(\tilde{\boldsymbol{w}}, \tilde{\vartheta}),(\boldsymbol{\varphi}, \psi)\rangle=\langle(\tilde{\boldsymbol{w}}, \tilde{\vartheta}), T(\boldsymbol{\varphi}, \psi)\rangle .
$$

On the other hand, we have both $\mathcal{S}_{[1-\ell-n / p]} \subset \boldsymbol{W}_{\ell+1,1}^{2, p}\left(\mathbb{R}^{n}\right) \times W_{\ell+1,1}^{1, p}\left(\mathbb{R}^{n}\right)$ and the imbedding $\boldsymbol{W}_{\ell+1,1}^{2, p}\left(\mathbb{R}^{n}\right) \times W_{\ell+1,1}^{1, p}\left(\mathbb{R}^{n}\right) \hookrightarrow \boldsymbol{W}_{\ell-1}^{0, p}\left(\mathbb{R}^{n}\right) \times W_{\ell-1}^{-1, p}\left(\mathbb{R}^{n}\right)$ under hypothesis (4.1), then by density of $\mathcal{D}\left(\mathbb{R}^{n}\right) \times \mathcal{D}\left(\mathbb{R}^{n}\right)$ in $\boldsymbol{W}_{\ell+1,1}^{2, p}\left(\mathbb{R}^{n}\right) \times W_{\ell+1,1}^{1, p}\left(\mathbb{R}^{n}\right)$, we can deduce that (4.3) holds for any $(\boldsymbol{\varphi}, \psi) \in \mathcal{S}_{[1-\ell-n / p]}$ and thus $T(\tilde{\boldsymbol{w}}, \tilde{\vartheta}) \perp \mathcal{S}_{[1-\ell-n / p]}$.

With this orthogonality condition, we can apply Theorem 1.3 and it follows that $(\tilde{\boldsymbol{w}}, \tilde{\vartheta}) \in \boldsymbol{W}_{-\ell+1}^{2, p^{\prime}}\left(\mathbb{R}^{n}\right) \times W_{-\ell+1}^{1, p^{\prime}}\left(\mathbb{R}^{n}\right)$. Since $\tilde{\boldsymbol{w}}$ and $\tilde{\vartheta}$ are the zero extensions of $\boldsymbol{w}$ and $\vartheta$, it follows that $(\boldsymbol{w}, \vartheta) \in \stackrel{\circ}{\boldsymbol{W}}_{-\ell+1}^{2, p^{\prime}}\left(\mathbb{R}_{+}^{n}\right) \times \stackrel{\circ}{W_{-\ell+1}^{1, p^{\prime}}}\left(\mathbb{R}_{+}^{n}\right)$. Then, by density of $\mathcal{D}\left(\mathbb{R}_{+}^{n}\right) \times$ $\mathcal{D}\left(\mathbb{R}_{+}^{n}\right)$ in $\stackrel{\circ}{\boldsymbol{W}}_{-\ell+p^{\prime}}^{2}\left(\mathbb{R}_{+}^{n}\right) \times \stackrel{\circ}{W_{-\ell+1}^{1, p^{\prime}}}\left(\mathbb{R}_{+}^{n}\right)$, we can construct a sequence $\left(\boldsymbol{w}_{k}, \vartheta_{k}\right)_{k \in \mathbb{N}} \subset$ $\mathcal{D}\left(\mathbb{R}_{+}^{n}\right) \times \mathcal{D}\left(\mathbb{R}_{+}^{n}\right)$ such that $\left(\boldsymbol{w}_{k}, \vartheta_{k}\right) \rightarrow(\boldsymbol{w}, \vartheta)$ in $\stackrel{\circ}{\boldsymbol{W}_{-\ell+1}^{2, p^{\prime}}}\left(\mathbb{R}_{+}^{n}\right) \times \stackrel{\circ}{W_{-\ell+1}^{1, p^{\prime}}}\left(\mathbb{R}_{+}^{n}\right)$. Thus, for any $(\boldsymbol{u}, \pi) \in T_{\ell, 1}^{p}\left(\mathbb{R}_{+}^{n}\right)$, we have

$$
\begin{aligned}
\langle\Lambda,(\boldsymbol{u}, \pi)\rangle & =-\langle T(\boldsymbol{w}, \vartheta),(\boldsymbol{u}, \pi)\rangle+\langle(\boldsymbol{w}, \vartheta), T(\boldsymbol{u}, \pi)\rangle \\
& =\lim _{k \rightarrow \infty}\left\{-\left\langle T\left(\boldsymbol{w}_{k}, \vartheta_{k}\right),(\boldsymbol{u}, \pi)\right\rangle+\left\langle\left(\boldsymbol{w}_{k}, \vartheta_{k}\right), T(\boldsymbol{u}, \pi)\right\rangle\right\} \\
& =0
\end{aligned}
$$


i.e. $\Lambda$ is identically zero.

Thanks to this density lemma, we can prove the following result:

LEMMA 4.2. Let $\ell \in \mathbb{Z}$. Under hypothesis (4.1), we can define the following linear continuous mapping - that is the traces of order 1 for the tangential component and of order 0 for the normal component of the velocity field - :

$$
\begin{aligned}
\gamma^{\sharp}: T_{\ell, 1}^{p}\left(\mathbb{R}_{+}^{n}\right) & \longrightarrow \boldsymbol{W}_{\ell-1}^{-1-1 / p, p}(\Gamma) \times W_{\ell-1}^{-1 / p, p}(\Gamma), \\
(\boldsymbol{u}, \pi) & \left.\longmapsto\left(\partial_{n} \boldsymbol{u}^{\prime}, u_{n}\right)\right|_{\Gamma}=\left(\gamma_{1} u_{1}, \ldots, \gamma_{1} u_{n-1}, \gamma_{0} u_{n}\right) .
\end{aligned}
$$

Moreover, we have the following Green formula:

$$
\begin{array}{r}
\forall(\boldsymbol{u}, \pi) \in T_{\ell, 1}^{p}\left(\mathbb{R}_{+}^{n}\right), \forall(\boldsymbol{\varphi}, \psi) \in \boldsymbol{W}_{-\ell+1}^{2, p^{\prime}}\left(\mathbb{R}_{+}^{n}\right) \times W_{-\ell+1}^{1, p^{\prime}}\left(\mathbb{R}_{+}^{n}\right) \\
\text { such that } \varphi_{n}=0, \partial_{n} \boldsymbol{\varphi}^{\prime}=\mathbf{0} \text { and } \operatorname{div} \boldsymbol{\varphi}=0 \text { on } \Gamma, \\
\langle T(\boldsymbol{u}, \pi),(\boldsymbol{\varphi}, \psi)\rangle_{\boldsymbol{W}_{\ell+1,1}^{0, p}\left(\mathbb{R}_{+}^{n}\right) \times W_{\ell, 1}^{0, p}\left(\mathbb{R}_{+}^{n}\right), \boldsymbol{W}_{-\ell-1,-1}^{0, p^{\prime}}\left(\mathbb{R}_{+}^{n}\right) \times W_{-\ell,-1}^{0, p^{\prime}}\left(\mathbb{R}_{+}^{n}\right)} \\
=\langle(\boldsymbol{u}, \pi), T(\boldsymbol{\varphi}, \psi)\rangle_{\boldsymbol{W}_{\ell-1}^{0, p}\left(\mathbb{R}_{+}^{n}\right) \times W_{\ell-1}^{-1, p}\left(\mathbb{R}_{+}^{n}\right), \boldsymbol{W}_{-\ell+1}^{0, p^{\prime}}\left(\mathbb{R}_{+}^{n}\right) \times{\stackrel{\circ}{-1, p^{\prime}}}_{-\ell}^{1,}\left(\mathbb{R}_{+}^{n}\right)} \\
+\left\langle\left(\partial_{n} \boldsymbol{u}^{\prime}, u_{n}\right),\left(\boldsymbol{\varphi}^{\prime}, \psi-\partial_{n} \varphi_{n}\right)\right\rangle_{\Gamma} .
\end{array}
$$

Proof. Let us make two remarks to start. Firstly, the left-hand term in (4.4) is nothing but the integral $\int_{\mathbb{R}_{+}^{n}} T(\boldsymbol{u}, \pi) \cdot(\boldsymbol{\varphi}, \psi) \mathrm{d} x$. Secondly, the reason for the logarithmic factor in the definition of $T_{\ell, 1}^{p}\left(\mathbb{R}_{+}^{n}\right)$ is that the imbeddings $\boldsymbol{W}_{-\ell+1}^{2, p^{\prime}}\left(\mathbb{R}_{+}^{n}\right) \hookrightarrow$ $\boldsymbol{W}_{-\ell-1,-1}^{0, p^{\prime}}\left(\mathbb{R}_{+}^{n}\right)$ and $W_{-\ell+1}^{1, p^{\prime}}\left(\mathbb{R}_{+}^{n}\right) \hookrightarrow W_{-\ell,-1}^{0, p^{\prime}}\left(\mathbb{R}_{+}^{n}\right)$ hold without supplementary critical value with respect to $(4.1)$ — whereas the imbedding $\boldsymbol{W}_{-\ell+1}^{2, p^{\prime}}\left(\mathbb{R}_{+}^{n}\right) \hookrightarrow \boldsymbol{W}_{-\ell-1}^{0, p^{\prime}}\left(\mathbb{R}_{+}^{n}\right)$ fails if $n / p^{\prime} \in\{\ell, \ell+1\}-$.

So we can write the following Green formula:

$$
\begin{array}{r}
\forall(\boldsymbol{u}, \pi) \in \mathcal{D}\left(\overline{\mathbb{R}_{+}^{n}}\right) \times \mathcal{D}\left(\overline{\mathbb{R}_{+}^{n}}\right), \forall(\boldsymbol{\varphi}, \psi) \in \boldsymbol{W}_{-\ell+1}^{2, p^{\prime}}\left(\mathbb{R}_{+}^{n}\right) \times W_{-\ell+1}^{1, p^{\prime}}\left(\mathbb{R}_{+}^{n}\right) \\
\text { such that } \varphi_{n}=0, \partial_{n} \boldsymbol{\varphi}^{\prime}=\mathbf{0} \text { and } \operatorname{div} \boldsymbol{\varphi}=0 \text { on } \Gamma, \\
\int_{\mathbb{R}_{+}^{n}} T(\boldsymbol{u}, \pi) \cdot(\boldsymbol{\varphi}, \psi) \mathrm{d} x=\int_{\mathbb{R}_{+}^{n}}(\boldsymbol{u}, \pi) \cdot T(\boldsymbol{\varphi}, \psi) \mathrm{d} x \\
+\int_{\Gamma}\left(\partial_{n} \boldsymbol{u}^{\prime}, u_{n}\right) \cdot\left(\boldsymbol{\varphi}^{\prime}, \psi-\partial_{n} \varphi_{n}\right) \mathrm{d} x^{\prime} .
\end{array}
$$

We can deduce the following estimate:

$$
\left|\left\langle\left(\partial_{n} \boldsymbol{u}^{\prime}, u_{n}\right),\left(\boldsymbol{\varphi}^{\prime}, \psi-\partial_{n} \varphi_{n}\right)\right\rangle_{\Gamma}\right| \leqslant\|(\boldsymbol{u}, \pi)\|_{T_{\ell, 1}^{p}\left(\mathbb{R}_{+}^{n}\right)}\|(\boldsymbol{\varphi}, \psi)\|_{\boldsymbol{W}_{-\ell+1}^{2, p^{\prime}}\left(\mathbb{R}_{+}^{n}\right) \times W_{-\ell+1}^{1, p^{\prime}\left(\mathbb{R}_{+}^{n}\right)}} .
$$

According to Lemma 1.1, for any $\boldsymbol{\mu}=\left(\boldsymbol{\mu}^{\prime}, \mu_{n}\right) \in \boldsymbol{W}_{-\ell+1}^{2-1 / p^{\prime}, p^{\prime}}(\Gamma) \times W_{-\ell+1}^{1-1 / p^{\prime}, p}(\Gamma)$, there exists a lifting function $(\boldsymbol{\varphi}, \psi) \in \boldsymbol{W}_{-\ell+1}^{2, p^{\prime}}\left(\mathbb{R}_{+}^{n}\right) \times W_{-\ell+1}^{1, p^{\prime}}\left(\mathbb{R}_{+}^{n}\right)$ such that

$$
\begin{aligned}
\left(\gamma_{0}, \gamma_{1}\right) \boldsymbol{\varphi}^{\prime} & =\left(\boldsymbol{\mu}^{\prime}, \mathbf{0}\right) \in \boldsymbol{W}_{-\ell+1}^{2-1 / p^{\prime}, p^{\prime}}(\Gamma) \times \boldsymbol{W}_{-\ell+1}^{1-1 / p^{\prime}, p}(\Gamma), \\
\left(\gamma_{0}, \gamma_{1}\right) \varphi_{n} & =\left(0,-\operatorname{div}^{\prime} \boldsymbol{\mu}^{\prime}\right) \in W_{-\ell+1}^{2-1 / p^{\prime}, p^{\prime}}(\Gamma) \times W_{-\ell+1}^{1-1 / p^{\prime}, p}(\Gamma), \\
\gamma_{0} \psi & =\mu_{n}-\operatorname{div}^{\prime} \boldsymbol{\mu}^{\prime} \in W_{-\ell+1}^{1-1 / p^{\prime}, p}(\Gamma)
\end{aligned}
$$

- i.e. $\left(\boldsymbol{\varphi}^{\prime}, \psi-\partial_{n} \varphi_{n}\right)=\boldsymbol{\mu}$ with $\varphi_{n}=0, \partial_{n} \boldsymbol{\varphi}^{\prime}=\mathbf{0}$ and $\operatorname{div} \boldsymbol{\varphi}=0$ on $\Gamma-$, satisfying

$$
\|(\boldsymbol{\varphi}, \psi)\|_{\boldsymbol{W}_{-\ell+1}^{2, p^{\prime}}\left(\mathbb{R}_{+}^{n}\right) \times W_{-\ell+1}^{1, p^{\prime}}\left(\mathbb{R}_{+}^{n}\right)} \leqslant C\|\boldsymbol{\mu}\|_{\boldsymbol{W}_{-\ell+1}^{2-1 / p^{\prime}, p^{\prime}}(\Gamma) \times W_{-\ell+1}^{1-1 / p^{\prime}, p^{\prime}}(\Gamma)},
$$


where $C$ is a constant not depending on $(\boldsymbol{\varphi}, \psi)$ and $\boldsymbol{\mu}$. Then we can deduce that

$$
\left\|\left(\partial_{n} \boldsymbol{u}^{\prime}, u_{n}\right)\right\|_{\boldsymbol{W}_{\ell-1}^{-1 / p, p}(\Gamma) \times W_{\ell-1}^{-1 / p, p}(\Gamma)} \leqslant C\|(\boldsymbol{u}, \pi)\|_{T_{\ell, 1}^{p}\left(\mathbb{R}_{+}^{n}\right)} .
$$

Thus the linear mapping $\gamma^{\sharp}:\left.(\boldsymbol{u}, \pi) \longmapsto\left(\partial_{n} \boldsymbol{u}^{\prime}, u_{n}\right)\right|_{\Gamma}$ defined on $\mathcal{D}\left(\overline{\mathbb{R}_{+}^{n}}\right) \times \mathcal{D}\left(\overline{\mathbb{R}_{+}^{n}}\right)$ is continuous for the norm of $T_{\ell, 1}^{p}\left(\mathbb{R}_{+}^{n}\right)$. In addition, since $\mathcal{D}\left(\overline{\mathbb{R}_{+}^{n}}\right) \times \mathcal{D}\left(\overline{\mathbb{R}_{+}^{n}}\right)$ is dense in $T_{\ell, 1}^{p}\left(\mathbb{R}_{+}^{n}\right)$, the mapping $\gamma^{\sharp}$ can be extended by continuity to a mapping still called $\gamma^{\sharp} \in \mathcal{L}\left(T_{\ell, 1}^{p}\left(\mathbb{R}_{+}^{n}\right) ; \boldsymbol{W}_{\ell-1}^{-1-1 / p, p}(\Gamma) \times W_{\ell-1}^{-1 / p, p}(\Gamma)\right)$. Moreover, we also can deduce the formula (4.4) from (4.5) by density of $\mathcal{D}\left(\overline{\mathbb{R}_{+}^{n}}\right) \times \mathcal{D}\left(\overline{\mathbb{R}_{+}^{n}}\right)$ in $T_{\ell, 1}^{p}\left(\mathbb{R}_{+}^{n}\right)$.

Thanks to this lemma, we now can give the result for singular boundary conditions.

Theorem 4.3. Let $\ell \in \mathbb{Z}$ with hypothesis (4.1). For any $\boldsymbol{g}=\left(\boldsymbol{g}^{\prime}, g_{n}\right) \in$ $\boldsymbol{W}_{\ell-1}^{-1-1 / p, p}(\Gamma) \times W_{\ell-1}^{-1 / p, p}(\Gamma)$, satisfying the compatibility condition

$$
\begin{aligned}
\forall \boldsymbol{\varphi}=\left(\boldsymbol{\varphi}^{\prime}, \varphi_{n}\right) \in \mathcal{N}_{\left[1+\ell-n / p^{\prime}\right]}^{\Delta} \times \mathcal{A}_{\left[1+\ell-n / p^{\prime}\right]}^{\Delta}, & \\
& \quad-\left\langle\boldsymbol{g}^{\prime}, \boldsymbol{\varphi}^{\prime}\right\rangle_{\boldsymbol{W}_{\ell-1}^{-1-1 / p, p}(\Gamma) \times \boldsymbol{W}_{-\ell+1}^{2-1 / p^{\prime}, p^{\prime}}(\Gamma)} \\
& \quad+\left\langle g_{n}, \partial_{n} \boldsymbol{\varphi}_{n}\right\rangle_{W_{\ell-1}^{-1 / p, p}(\Gamma) \times W_{-\ell+1}^{1-1 / p^{\prime}, p^{\prime}}(\Gamma)}=0
\end{aligned}
$$

problem (2.1) admits a solution $(\boldsymbol{u}, \pi) \in \boldsymbol{W}_{\ell-1}^{0, p}\left(\mathbb{R}_{+}^{n}\right) \times W_{\ell-1}^{-1, p}\left(\mathbb{R}_{+}^{n}\right)$, unique up to an element of $\mathcal{S}_{[1-\ell-n / p]}^{\sharp}$, and there exists a constant $C$ such that

$$
\inf _{(\boldsymbol{\lambda}, \mu) \in \mathcal{S}_{[1-\ell-n / p]}^{\sharp}}\left(\|\boldsymbol{u}+\boldsymbol{\lambda}\|_{\boldsymbol{W}_{\ell-1}^{0, p}\left(\mathbb{R}_{+}^{n}\right)}+\|\pi+\mu\|_{W_{\ell-1}^{-1, p}\left(\mathbb{R}_{+}^{n}\right)}\right) \leqslant C\|\boldsymbol{g}\|_{\Gamma} .
$$

Proof. To start with, let us observe that such a pair $(\boldsymbol{u}, \pi)$ belongs to $T_{\ell, 1}^{p}\left(\mathbb{R}_{+}^{n}\right)$ and then Lemma 4.2 gives a sense to these boundary conditions. Next, we can observe that problem (2.1) is equivalent to the variational formulation: Find $(\boldsymbol{u}, \pi) \in \boldsymbol{W}_{\ell-1}^{0, p}\left(\mathbb{R}_{+}^{n}\right) \times$ $W_{\ell-1}^{-1, p}\left(\mathbb{R}_{+}^{n}\right)$ satisfying

$$
\begin{aligned}
& \forall(\boldsymbol{v}, \vartheta) \in \boldsymbol{W}_{-\ell+1}^{2, p^{\prime}}\left(\mathbb{R}_{+}^{n}\right) \times W_{-\ell+1}^{1, p^{\prime}}\left(\mathbb{R}_{+}^{n}\right) \\
& \quad \text { such that } v_{n}=0, \partial_{n} \boldsymbol{v}^{\prime}=\mathbf{0} \text { and } \operatorname{div} \boldsymbol{v}=0 \text { on } \Gamma, \\
& \langle(\boldsymbol{u}, \pi), T(\boldsymbol{v}, \vartheta)\rangle_{\boldsymbol{W}_{\ell-1}^{0, p}\left(\mathbb{R}_{+}^{n}\right) \times W_{\ell-1}^{-1, p}\left(\mathbb{R}_{+}^{n}\right), \boldsymbol{W}_{-\ell+1}^{0, p^{\prime}}\left(\mathbb{R}_{+}^{n}\right) \times \stackrel{\circ}{W}_{-\ell+1}^{1, p^{\prime}}\left(\mathbb{R}_{+}^{n}\right)}= \\
& -\left\langle\boldsymbol{g},\left(\boldsymbol{v}^{\prime}, \vartheta-\partial_{n} v_{n}\right)\right\rangle_{\boldsymbol{W}_{\ell-1}^{-1-1 / p, p}(\Gamma) \times W_{\ell-1}^{-1 / p, p}(\Gamma), \boldsymbol{W}_{-\ell+1}^{2-1 / p^{\prime}, p^{\prime}}(\Gamma) \times W_{-\ell+1}^{1 / p, p^{\prime}}(\Gamma)} .
\end{aligned}
$$

Indeed, the direct implication is straightforward. Conversely, if the pair $(\boldsymbol{u}, \pi)$ satisfies (4.7) then we have for any $(\varphi, \psi) \in \mathcal{D}\left(\mathbb{R}_{+}^{n}\right) \times \mathcal{D}\left(\mathbb{R}_{+}^{n}\right)$,

$$
\langle T(\boldsymbol{u}, \pi),(\boldsymbol{\varphi}, \psi)\rangle_{\mathcal{D}^{\prime}\left(\mathbb{R}_{+}^{n}\right) \times \mathcal{D}^{\prime}\left(\mathbb{R}_{+}^{n}\right), \mathcal{D}\left(\mathbb{R}_{+}^{n}\right) \times \mathcal{D}\left(\mathbb{R}_{+}^{n}\right)}=\langle(\boldsymbol{u}, \pi), T(\boldsymbol{\varphi}, \psi)\rangle=0,
$$

thus $T(\boldsymbol{u}, \pi)=\mathbf{0}$ in $\mathbb{R}_{+}^{n}$. In addition, according to the Green formula (4.4), we have

$$
\begin{aligned}
& \forall(\boldsymbol{v}, \vartheta) \in \boldsymbol{W}_{-\ell+1}^{2, p^{\prime}}\left(\mathbb{R}_{+}^{n}\right) \times W_{-\ell+1}^{1, p^{\prime}}\left(\mathbb{R}_{+}^{n}\right) \\
& \text { such that } v_{n}=0, \partial_{n} \boldsymbol{v}^{\prime}=\mathbf{0} \text { and } \operatorname{div} \boldsymbol{v}=0 \text { on } \Gamma \\
&\left\langle\left(\partial_{n} \boldsymbol{u}^{\prime}, u_{n}\right),\left(\boldsymbol{v}^{\prime}, \vartheta-\partial_{n} v_{n}\right)\right\rangle_{\Gamma}=\left\langle\boldsymbol{g},\left(\boldsymbol{v}^{\prime}, \vartheta-\partial_{n} v_{n}\right)\right\rangle_{\Gamma}
\end{aligned}
$$


As we saw in the proof of Lemma 4.2 , by Lemma 1.1, it follows that for any $\boldsymbol{\mu} \in$ $\boldsymbol{W}_{-\ell+1}^{2-1 / p^{\prime}, p^{\prime}}(\Gamma) \times W_{-\ell+1}^{1-1 / p^{\prime}, p^{\prime}}(\Gamma)$,

$$
\left\langle\left(\partial_{n} \boldsymbol{u}^{\prime}-\boldsymbol{g}^{\prime}, u_{n}-g_{n}\right), \boldsymbol{\mu}\right\rangle_{\Gamma}=0
$$

that is $\partial_{n} \boldsymbol{u}^{\prime}=\boldsymbol{g}^{\prime}$ and $u_{n}=g_{n}$ on $\Gamma$. Hence $(\boldsymbol{u}, \pi)$ satisfies $(2.1)$.

Now, let us solve problem (4.7). By Theorem 3.10, we know that under hypothesis (4.1), for all $(\boldsymbol{f}, h) \in \boldsymbol{W}_{-\ell+1}^{0, p^{\prime}}\left(\mathbb{R}_{+}^{n}\right) \times \stackrel{\circ}{W_{-\ell+1}^{1, p^{\prime}}}\left(\mathbb{R}_{+}^{n}\right) \perp \mathcal{S}_{[1-\ell-n / p]}^{\sharp}$, there exists a unique $(\boldsymbol{v}, \vartheta) \in \boldsymbol{W}_{-\ell+1}^{2, p^{\prime}}\left(\mathbb{R}_{+}^{n}\right) \times W_{-\ell+1}^{1, p^{\prime}}\left(\mathbb{R}_{+}^{n}\right) / \mathcal{S}_{\left[1+\ell-n / p^{\prime}\right]}^{\sharp}$ solution to

$$
-\Delta \boldsymbol{v}+\nabla \vartheta=\boldsymbol{f} \text { and } \operatorname{div} \boldsymbol{v}=h \text { in } \mathbb{R}_{+}^{n}, \quad \partial_{n} \boldsymbol{v}^{\prime}=\mathbf{0} \text { and } v_{n}=0 \text { on } \Gamma,
$$

with the estimate

$$
\|(\boldsymbol{v}, \vartheta)\|_{\boldsymbol{W}_{-\ell+1}^{2, p^{\prime}}\left(\mathbb{R}_{+}^{n}\right) \times W_{-\ell+1}^{1, p^{\prime}}\left(\mathbb{R}_{+}^{n}\right) / \mathcal{S}_{\left[1+\ell-n / p^{\prime}\right]}^{\sharp}} \leqslant C\left(\|\boldsymbol{f}\|_{\boldsymbol{W}_{-\ell+1}^{0, p^{\prime}\left(\mathbb{R}_{+}^{n}\right)}}+\|h\|_{W_{-\ell+1}^{1, p^{\prime}\left(\mathbb{R}_{+}^{n}\right)}}\right) .
$$

Consider the linear form $\Xi:(\boldsymbol{f}, h) \longmapsto\left\langle\boldsymbol{g},\left(\boldsymbol{v}^{\prime}, \vartheta-\partial_{n} v_{n}\right)\right\rangle_{\Gamma}$ defined on the product space $\boldsymbol{W}_{-\ell+1}^{0, p^{\prime}}\left(\mathbb{R}_{+}^{n}\right) \times \stackrel{\circ}{W_{-\ell+1}^{1, p^{\prime}}}\left(\mathbb{R}_{+}^{n}\right) \perp \mathcal{S}_{[1-\ell-n / p]}^{\sharp}$. According to (4.6), we have for any $\boldsymbol{\varphi} \in \mathcal{N}_{\left[1+\ell-n / p^{\prime}\right]}^{\Delta} \times \mathcal{A}_{\left[1+\ell-n / p^{\prime}\right]}^{\Delta}$ - or equivalently, for any $(\boldsymbol{\lambda}, \mu) \in \mathcal{S}_{\left[1+\ell-n / p^{\prime}\right]}^{\sharp}$ 一,

$$
\begin{aligned}
& |\Xi(\boldsymbol{f}, h)|=\left|\left\langle\boldsymbol{g},\left(\boldsymbol{v}^{\prime}+\varphi^{\prime}, \vartheta-\partial_{n} v_{n}+\partial_{n} \varphi_{n}\right)\right\rangle_{\Gamma}\right| \\
& =\left|\left\langle\boldsymbol{g},\left(\left[\boldsymbol{v}^{\prime}+\boldsymbol{\lambda}^{\prime}\right],[\vartheta+\mu]-\partial_{n}\left[v_{n}+\lambda_{n}\right]\right)\right\rangle_{\Gamma}\right| \\
& \leqslant C\|\boldsymbol{g}\|_{\boldsymbol{W}_{\ell-1}^{-1-1 / p, p}(\Gamma) \times W_{\ell-1}^{-1 / p, p}(\Gamma)}\|(\boldsymbol{v}, \vartheta)+(\boldsymbol{\lambda}, \mu)\|_{\boldsymbol{W}_{-\ell+1}^{2, p^{\prime}}\left(\mathbb{R}_{+}^{n}\right) \times W_{-\ell+1}^{1, p^{\prime}}\left(\mathbb{R}_{+}^{n}\right)} \text {. }
\end{aligned}
$$

Thus

$$
\begin{aligned}
|\Xi(\boldsymbol{f}, h)| & \leqslant C\|\boldsymbol{g}\|_{\boldsymbol{W}_{\ell-1}^{-1-1 / p, p}(\Gamma) \times W_{\ell-1}^{-1 / p, p}(\Gamma)}\|(\boldsymbol{v}, \vartheta)\|_{\boldsymbol{W}_{-\ell+1}^{2, p^{\prime}}\left(\mathbb{R}_{+}^{n}\right) \times W_{-\ell+1}^{1, p^{\prime}\left(\mathbb{R}_{+}^{n}\right) / \mathcal{S}_{\left[1+\ell-n / p^{\prime}\right]}^{\sharp}}} \\
& \leqslant C\|\boldsymbol{g}\|_{\boldsymbol{W}_{\ell-1}^{-1-1 / p, p}(\Gamma) \times W_{\ell-1}^{-1 / p, p}(\Gamma)}\left(\|\boldsymbol{f}\|_{\boldsymbol{W}_{-\ell+1}^{0, p^{\prime}}\left(\mathbb{R}_{+}^{n}\right)}+\|h\|_{W_{-\ell+1}^{1, p^{\prime}}\left(\mathbb{R}_{+}^{n}\right)}\right) .
\end{aligned}
$$

In other words, $\Xi$ is continuous on $\boldsymbol{W}_{-\ell+1}^{0, p^{\prime}}\left(\mathbb{R}_{+}^{n}\right) \times \stackrel{\circ}{{ }^{1}}{ }_{-\ell+1}^{1, p^{\prime}}\left(\mathbb{R}_{+}^{n}\right) \perp \mathcal{S}_{[1-\ell-n / p]}^{\sharp}$, and according to the Riesz representation theorem, we can deduce that there exists a unique $(\boldsymbol{u}, \pi) \in \boldsymbol{W}_{\ell-1}^{0, p}\left(\mathbb{R}_{+}^{n}\right) \times W_{\ell-1}^{-1, p}\left(\mathbb{R}_{+}^{n}\right) / \mathcal{S}_{[1-\ell-n / p]}^{\sharp}$ - which is the dual space of $\boldsymbol{W}_{-\ell+1}^{0, p^{\prime}}\left(\mathbb{R}_{+}^{n}\right) \times \stackrel{\circ}{W_{-\ell+1}^{1, p^{\prime}}}\left(\mathbb{R}_{+}^{n}\right) \perp \mathcal{S}_{[1-\ell-n / p]}^{\sharp}$-, such that

$$
\begin{aligned}
& \forall(\boldsymbol{f}, h) \in \boldsymbol{W}_{-\ell+1}^{0, p^{\prime}}\left(\mathbb{R}_{+}^{n}\right) \times \stackrel{\circ}{W_{-\ell+1}^{1, p^{\prime}}}\left(\mathbb{R}_{+}^{n}\right), \\
& \Xi(\boldsymbol{f}, h)=\langle\boldsymbol{u}, \boldsymbol{f}\rangle_{\boldsymbol{W}_{\ell-1}^{0, p}\left(\mathbb{R}_{+}^{n}\right) \times \boldsymbol{W}_{-\ell+1}^{0, p^{\prime}}\left(\mathbb{R}_{+}^{n}\right)}+\langle\pi,-h\rangle_{W_{\ell-1}^{-1, p}\left(\mathbb{R}_{+}^{n}\right) \times \stackrel{\circ}{W}_{-\ell+1}^{1, p^{\prime}}\left(\mathbb{R}_{+}^{n}\right)} .
\end{aligned}
$$

Then, we can conclude that the pair $(\boldsymbol{u}, \pi)$ satisfies (4.7) and moreover the kernel of the associated operator is $\mathcal{S}_{[1-\ell-n / p]}^{\sharp}$.

To end this study, using the method of the proof of the existence in Theorem 3.5, we can establish the existence of very weak solutions to the nonhomogeneous problem with very singular boundary conditions.

THEOREM 4.4. Let $\ell \in \mathbb{Z}$ and assume that

$$
n / p^{\prime} \notin\{1, \ldots, \ell+1\} \quad \text { and } \quad n / p \notin\{1, \ldots,-\ell+1\} .
$$


For any $\boldsymbol{f} \in \boldsymbol{W}_{\ell+1}^{0, p}\left(\mathbb{R}_{+}^{n}\right), h \in W_{\ell+1}^{1, p}\left(\mathbb{R}_{+}^{n}\right), g_{n} \in W_{\ell-1}^{-1 / p, p}(\Gamma), \boldsymbol{g}^{\prime} \in \boldsymbol{W}_{\ell-1}^{-1-1 / p, p}(\Gamma)$, satisfying the compatibility condition

$$
\begin{aligned}
& \forall \boldsymbol{\varphi} \in \mathcal{N}_{\left[1+\ell-n / p^{\prime}\right]}^{\Delta} \times \mathcal{A}_{\left[1+\ell-n / p^{\prime}\right]}^{\Delta}, \\
& \qquad \begin{aligned}
\int_{\mathbb{R}_{+}^{n}}(\boldsymbol{f}-\nabla h) \cdot \boldsymbol{\varphi} \mathrm{d} x+\left\langle\operatorname{div} \boldsymbol{f}, \Pi_{N} \operatorname{div} \boldsymbol{\varphi}\right\rangle_{W_{\ell+1}^{-1, p}\left(\mathbb{R}_{+}^{n}\right) \times \stackrel{\circ}{W}_{-\ell-1}^{1, p^{\prime}}\left(\mathbb{R}_{+}^{n}\right)} \\
+\left\langle g_{n}, \partial_{n} \varphi_{n}\right\rangle_{\boldsymbol{W}_{\ell-1}^{-1 / p, p}(\Gamma) \times \boldsymbol{W}_{-\ell+1}^{1-1 / p^{\prime}, p^{\prime}}(\Gamma)} \\
\quad-\left\langle\boldsymbol{g}^{\prime}, \boldsymbol{\varphi}^{\prime}\right\rangle_{\boldsymbol{W}_{\ell-1}^{-1-1 / p, p}(\Gamma) \times \boldsymbol{W}_{-\ell+1}^{2-1 / p^{\prime}, p^{\prime}}(\Gamma)}=0
\end{aligned}
\end{aligned}
$$

problem $\left(S^{\sharp}\right)$ admits a solution $(\boldsymbol{u}, \pi) \in \boldsymbol{W}_{\ell+1}^{0, p}\left(\mathbb{R}_{+}^{n}\right) \times W_{\ell-1}^{-1, p}\left(\mathbb{R}_{+}^{n}\right)$, unique up to an element of $\mathcal{S}_{[1-\ell-n / p]}^{\sharp}$, with the estimate

$$
\begin{aligned}
& \inf _{(\boldsymbol{\lambda}, \mu) \in \mathcal{S}_{[1-\ell-n / p]}^{\sharp}}\left(\|\boldsymbol{u}+\boldsymbol{\lambda}\|_{\boldsymbol{W}_{\ell-1}^{0, p}\left(\mathbb{R}_{+}^{n}\right)}+\|\pi+\mu\|_{W_{\ell-1}^{-1, p}\left(\mathbb{R}_{+}^{n}\right)}\right) \leqslant \\
& C\left(\|\boldsymbol{f}\|_{\boldsymbol{W}_{\ell+1}^{0, p}\left(\mathbb{R}_{+}^{n}\right)}+\|h\|_{W_{\ell+1}^{0, p}\left(\mathbb{R}_{+}^{n}\right)}+\left\|g_{n}\right\|_{W_{\ell-1}^{-1 / p, p}(\Gamma)}+\left\|\boldsymbol{g}^{\prime}\right\|_{\boldsymbol{W}_{\ell-1}^{-1-1 / p, p}(\Gamma)}\right) .
\end{aligned}
$$

Acknowledgements. The work of S. Nečasová was supported by the Academy of Sciences of the Czech Republic, Institutional Research Plan No. AV0Z10190503 and by the Grant Agency of the Academy of Sciences No. IAA1001190804. Also a part of work was done during her stay in Université de Pau et des Pays de l'Adour. She would like to express her gratitude to Prof. Amrouche and his colleagues for their extraordinary hospitality.

\section{REFERENCES}

[1] F. Alliot, C. Amrouche, The Stokes problem in $\mathbb{R}^{n}$ : an approach in weighted Sobolev spaces, Math. Meth. Appl. Sci., 9 (5) (1999), 723-754.

[2] C. Amrouche, V. Girault, J. Giroire, Weighted Sobolev spaces for Laplace's equation in $\mathbb{R}^{n}$, J. Math. Pures Appl., 73 (6) (1994), 579-606.

[3] C. Amrouche, S. NeČasová, Laplace equation in the half-space with a nonhomogeneous Dirichlet boundary condition, Mathematica Bohemica, 126 (2) (2001), 265-274.

[4] C. Amrouche, The Neumann problem in the half-space, C. R. Acad. Sci. Paris, Sér. I 335 (2002), 151-156.

[5] C. Amrouche, Y. Raudin, Nonhomogeneous biharmonic problem in the half-space, $L^{p}$ theory and generalized solutions, J. Differential Equations, 236 (2007), 57-81.

[6] C. Amrouche, Y. Raudin, Singular boundary conditions and regularity for the biharmonic problem in the half-space, Comm. Pure Appl. Anal., 6 (4) (2007), 957-982.

[7] C. Amrouche, S. NeČasová, Y. Raudin, Very weak, generalized and strong solutions to the Stokes system in the half-space, J. Differential Equations, 244 (2008), 887-915.

[8] A. Babin, A. Mahalov, B. Nicolaenko, 3D Navier-Stokes and Euler equations with initial data characterized by uniformly large vorticity, Dedicated to Professors Ciprian Foias and Roger Temam, Indiana Univ. Math. J., 50 (2001), Special Issue, 1-35.

[9] A. Babin, A. Mahalov, B. Nicolaenko, Global regularity of 3 D rotating Navier- Stokes equations for resonant domains, Appl. Math. Lett., 13 (4) (2000), 51-57.

[10] A. Babin, A. Mahalov, B. Nicolaenko, Global regularity of 3 D rotating Navier-Stokes equations for resonant domains, Indiana Univ. Math. J., 48 (3) (1999), 1133-1176.

[11] T. Z. Boulmezaoud, On the Stokes system and the biharmonic equation in the half-space: an approach via weighted Sobolev spaces, Math. Meth. Appl. Sci., 25 (2002), 373-398.

[12] L. Cattabriga, Su un problema al contorno relativo al sistema di equazioni di Stokes, Rend. Sem. Mat. Univ. Padova, 31 (1961), 308-340. 
[13] D. Einzel, P. Panzer, M. Liu, Boundary Condition for Fluid Flow: Curved or Rough Surfaces, Phys. Rev. Lett., 64(19), 2269-2272 (1990).

[14] R. FARwig, A Note on the Reflection Principle for the Biharmonic Equation and the Stokes System, Acta appl. Math., 25 (1994), 41-51.

[15] R. Farwig, H. Sohr, On the Stokes and Navier-Stokes System for Domains with Noncompact Boudary in $L^{q}$-spaces, Math. Nachr., 170 (1994), 53-77.

[16] G. P. GALDI, An introduction to the mathematical theory of the Navier-Stokes equations, vol. I and vol. II, Springer tracts in natural philosophy, 1994.

[17] B. Hanouzet, Espaces de Sobolev avec poids. Application au problème de Dirichlet dans un demi-espace, Rend. Sem. Mat. Univ. Padova, 46 (1971), 227-272.

[18] W. JäGER, A. MikeLIĆ, On the roughness- induced effective boundary conditions for an incompressible viscous flow, J. Differential Equations, 170 (2001), 96-122.

[19] O. A. Ladyzhenskaya, V. A. Solonnikov, Solution of some non-stationary problems of magnetohydrodynamics for a viscous incompressible fluid, Trudy Mat. Inst. Steklov, 59 (1960), 115-173.

[20] E. Lauga, M. Brenner, H. Stone, Microfluidics: The No-Slip Boundary Condition, Springer Handbook of Experimental Fluid Dynamics, Springer-Verlag 2007.

[21] V. G. Maz'Ya, B. A. Plamenevskil̆, L. I. Stupyalis, The three-dimentional problem of steady-state motion of a fluid with a free surface, in: Amer. Math. Soc. Transl., vol. 123 (1984), pp. 171-268.

[22] N. V. Priezjev, A. A. Darhuber, S. M. Troian, Slip behaviour in liquid films on surfaces of patterned wettability: Comparison between continuum and molecular dynamics simulations, Phys. Rev. E 71, 041608 (2005).

[23] N. V. Priezjev, S. M. Troian, Influence of periodic wall roughness on the slip behaviour at liquid/solid interfaces: molecular-scale simulations versus continuum predictions, J. Fluid Mech., 554 (2006), 25-46.

[24] T. QIAN, X.-P. WANG, P. Sheng, Hydrodynamic slip boundary condition at chemically patterned surfaces: A continuum deduction from molecular dynamics, Phys. Rev. E 72, 022501 (2005).

[25] N. TANAKA, On the boundary value problem for the stationary Stokes system in the half-space, J. Differential Equations, 115 (1995), 70-74.

[26] W. M. ZajaçCZKowski, Global special regular solutions to the Navier-Stokes equations in axially symmetric domains under boundary slip conditions, Dissertationes Math. (Rozprawy Mat.), 432 (2005), 138pp.

[27] Y. Zhu, S. Granick, Limits of the Hydrodynamic No-Slip Boundary Condition, Phys. Rev. Lett., 88(10), $106102(2002)$. 